\title{
An optimal transportation problem with a cost given by the Euclidean distance plus import/export taxes on the boundary
}

\author{
José M. Mazón, Julio D. Rossi and Julián Toledo
}

\begin{abstract}
In this paper we analyze a mass transportation problem in a bounded domain in which there is the possibility of import/export mass across the boundary paying a tax in addition to the transport cost that is assumed to be given by the Euclidean distance. We show a general duality argument and for the dual problem we find a Kantorovich potential as the limit as $p \rightarrow \infty$ of solutions to $p$-Laplacian type problems with nonlinear boundary conditions. In addition, we show that this limit encodes all the relevant information for our problem. It provides the masses that are exported and imported from the boundary and also allows the construction of an optimal transport plan. Finally we show that the arguments can be adapted to deal with the case in which the mass that can be exported/imported is bounded by prescribed functions.
\end{abstract}

\section{Introduction.}

Mass transport problems have been widely considered in the literature recently. This is due not only to their relevance for applications but also because of the novelty of the methods needed for their solution. The origin of such problems is an article from 1781 by Gaspard Monge, Mémoire su la théorie des déblais et des remblais, where he formulated a natural question in economics which deals with the optimal way of moving a mass distribution from one location to another so that the total work done is minimized. Here the cost of moving one unit of mass from $x$ to $y$ is measured with the Euclidean distance and the total work done is the sum (integral) of the transport cost, $|x-y|$, times the mass that is moved from $x$ to $y$. Evans and Gangbo in [7] used a PDE approach to prove the existence of an optimal transport map for the classical Monge problem, different to the first one given by Sudakov in 1979 using probabilistic methods ([11]; see also [2] and [4]). For general reference on transport problems we refer to [13] and [14].

Mathematics Subject Classification (2010): Primary 49J20,49J45; Secondary 45G10.

Keywords: Mass transport, Monge-Kantorovich problems, $p$-Laplacian equation. 
The result by Evans and Gangbo was the first motivation for the present work. Let $\Omega$ be an open bounded domain of $\mathbb{R}^{N}$. Although it is not necessary for our mathematical results, we will assume $\Omega$ is convex, because this is more convenient for the transport interpretation. Alternatively, one can use the geodesic distance inside the domain as the transport cost, but we prefer to restrict ourselves to the Euclidean distance to avoid technicalities that may obscure the main arguments. Let $f \in L^{\infty}(\Omega)$ and $N<p<+\infty$. Given $g_{i} \in C(\partial \Omega)$, with $g_{1} \leq g_{2}$ on $\partial \Omega$, we set

$$
W_{g_{1}, g_{2}}^{1, p}(\Omega)=\left\{u \in W^{1, p}(\Omega): g_{1} \leq u \leq g_{2} \text { on } \partial \Omega \text { in the sense of traces }\right\}
$$

and consider the functional

$$
\Psi_{p}(u):=\int_{\Omega} \frac{|\nabla u(x)|^{p}}{p} d x-\int_{\Omega} f(x) u(x) d x .
$$

Since $W_{g_{1}, g_{2}}^{1, p}(\Omega)$ is a closed convex subset of $W^{1, p}(\Omega)$ and the functional $\Psi_{p}$ is convex, lower semicontinuous, and coercive, the variational problem

$$
\min _{u \in W_{g_{1}, g_{2}}^{1, p}(\Omega)} \Psi_{p}(u)
$$

has a minimizer $u_{p}$ in $W_{g_{1}, g_{2}}^{1, p}(\Omega)$, which is a least energy solution of the obstacle problem

$$
\begin{cases}-\Delta_{p} u=f & \text { in } \Omega \\ g_{1} \leq u \leq g_{2} & \text { on } \partial \Omega\end{cases}
$$

This minimizer is unique in the case $\int_{\Omega} f \neq 0$; in the case $\int_{\Omega} f=0$, there can be multiple minimizers, but any two of them differ by a constant and this can happen only if there are two different constant functions between $g_{1}$ and $g_{2}$. Note that when every minimizer coincides with $g_{1}$ on some part of the boundary and with $g_{2}$ on another part of the boundary, then the minimizer is unique.

Let us also assume that $g_{1}$ and $g_{2}$ satisfy the following condition:

$$
g_{1}(x)-g_{2}(y) \leq|x-y| \quad \forall x, y \in \partial \Omega .
$$

Under this assumption we can take the limit as $p \rightarrow \infty$, see Theorem 3.1 , and obtain that $u_{p} \rightarrow u_{\infty}$ uniformly, and that the limit $u_{\infty}$ is a maximizer of the variational problem

$$
\max \left\{\int_{\Omega} w(x) f(x) d x: w \in W_{g_{1}, g_{2}}^{1, \infty}(\Omega),\|\nabla w\|_{L^{\infty}(\Omega)} \leq 1\right\} .
$$

At this point it is natural to ask the following question, that constitutes the main problem addressed in this paper:

Main problem: Let $f_{+}$and $f_{-}$be the positive and negative parts of $f$; that is $f=f_{+}-f_{-}$, with $f_{ \pm}$being $L^{\infty}$ masses. Can $u_{\infty}$, the limit of the sequence $u_{p}$, that is a maximizer for (1.4), be interpreted as a kind of Kantorovich potential for some transport problem involving $f_{+}$and $f_{-}$? 
The answer to this question is affirmative. Let us explain briefly and informally the mass transport problem that is related to this limit procedure; see $\S 2.2$ for more details. We have to transport some amount of material represented by $f_{+}$in $\Omega$ ( $f_{+}$encodes the amount of material and its location) to a hole with a distribution given by $f_{-}$also defined in $\Omega$. The goal is to transport all the mass (the penalty for not transporting the total mass is an infinite cost) $f_{+}$to $f_{-}$or to the boundary (exporting the mass out of $\Omega$ ). In doing this, we pay the transport costs given by the Euclidean distance $c(x, y)=|x-y|$ and when a unit of mass is left on a point $y \in \partial \Omega$ an additional cost given by $T_{e}(y)$, the export taxes. We also have the constraint of filling the hole completely (there is also an infinite-cost penalty for not covering all the mass in $\left.f_{-}\right)$, that is, we have to import product, if necessary, from the exterior of $\Omega$ (paying the transport costs plus the extra cost $T_{i}(x)$, the import taxes, for each unit of mass that enters $\Omega$ at the point $x \in \partial \Omega$ ). We have the freedom to chose to export or import mass provided we transport all the mass in $f_{+}$and cover all the mass of $f_{-}$. The main goal here is to minimize the total cost of this operation, that is given by the transport cost plus export/import taxes. Note that in this transport problem there appear two masses on $\partial \Omega$ that are unknowns (the ones that encode the mass that is exported and the mass that is imported). Also note that the usual mass balance condition

$$
\int_{\Omega} f_{+}(x) d x=\int_{\Omega} f_{-}(y) d y
$$

is not imposed since we can import or export mass through the boundary if necessary. This means that we can use $\partial \Omega$ as an infinite reserve/repository, we can take as much mass as we wish from the boundary, or send back as much mass as we want, provided we pay the transportation cost plus the import/export taxes.

Our ideas can be adapted to deal with a more realistic situation. With a cost such as that described above (the Euclidean distance plus import/export taxes) we can impose the restriction of not exceeding the pointwise quantity $M_{e}(x)$ (that we assume to be nonnegative) when we export some mass through $x \in \partial \Omega$ and we can also impose a pointwise restriction $M_{i}(x)$ (which is also assumed nonnegative) for import from $\partial \Omega$. Thus, in this case we do not assume that the boundary is an infinite reserve/repository, but we bound the quantities that can be exported or imported. For doing this the natural constraint that must be satisfied is given by

$$
-\int_{\partial \Omega} M_{e} \leq-\int_{\Omega}\left(f_{+}-f_{-}\right) \leq \int_{\partial \Omega} M_{i} .
$$

This says that one can transport all the positive mass in $f_{+}$and also that one can satisfy all the consumer demand (covering the whole of $f_{-}$). Hence the mass transport problem is feasible, and the problem becomes, as before, to minimize the cost. Two limit situations are as follows. When $T_{i}=T_{e}=0$ we have limited importation/exportation but without taxes. On the other hand, by assuming $M_{i}(x)=0$ or $M_{e}(x)=0$ on certain zones of the boundary, we preclude importation or exportation in these zones; if we impose these conditions on the whole boundary 
(assuming $\int_{\Omega} f_{+}=\int_{\Omega} f_{-}$) we obtain again a solution to the classical MongeKantorovich mass transport problem. This was solved by Evans and Gangbo by taking limits of $p$-Laplacian problems with homogeneous Dirichlet boundary conditions in a large ball. For simplicity and since the main mathematical difficulties are present without restricting the mass that can be exported/imported through the boundary, we present the details for this case and at the end of the article we sketch the necessary changes and adaptations that are needed to deal with the more realistic case.

A variant of this transport problem (allowing the possibility of import/export mass from/to $\partial \Omega$ ) was recently proposed in [9]. In [9] the transport cost is given by $|x-y|^{2}$ (which is strictly convex) with zero taxes on the boundary. The authors use this transport problem to define a new distance between measures and study the gradient flow of a particular entropy that coincides with the heat equation, with Dirichlet boundary condition equal to 1 (see [1] and [3] for related results concerning the relation between flows and transport problems). Here we deal with the cost given by the Euclidean distance $|x-y|$ (which is not strictly convex) and allow for nontrivial import/export taxes. In addition, we perform an approximation procedure using the $p$-Laplacian (as was done by Evans and Gangbo), something that is not needed for a quadratic cost. See also [6] and [8] for regularity results for a partial mass transport problem in which there is no boundary involved but the amount of mass that has to be transported is prescribed (here there is also considered a quadratic transport cost, $\left.|x-y|^{2}\right)$.

We briefly summarize the contents of this paper. In $\S 2$ we recall some well known facts, terminology, and notations related to the usual Monge-Kantorovich problem and its dual formulation, and, in $\S 2.2$, we describe the mass transport problem in which we are interested and study its dual formulation. The next section is devoted to obtaining the Kantorovich potential as a limit of the solutions of some obstacle problem associated with the $p$-Laplacian operator, to giving a complete proof of the duality, to obtaining the import/export masses from those $p$-Laplacian problems, and to showing how to construct optimal transport plans via optimal transport maps. In $\S 4$, to illustrate our results, we give some simple examples in which the solution to the mass transport problem described in $\$ 2.2$ can be explicitly computed. Finally in $\S 5$ we deal with the case of limited importation/exportation.

\section{Statement of the mass transport problem}

To state the problem more precisely we need some notation. Given a Borel subset $X \subset \mathbb{R}^{N}$, let $\mathcal{M}(X)$ denote the space of nonnegative Borel measures on $X$ with finite total mass. A measure $\gamma \in \mathcal{M}(X)$ and a Borel map $T: X \rightarrow \mathbb{R}^{N}$ induce a Borel measure $T \# \gamma$, the pushforward measure of $\gamma$ via $T$, defined by $(T \# \gamma)[B]=$ $\gamma\left[T^{-1}(B)\right]$. When we write $T \# f=g$, where $f$ and $g$ are nonnegative functions, this means that the measure having density $f$ is pushed forward to the measure having density $g$. 


\subsection{Mass transport theory}

The Monge problem. Given two measures $\gamma_{1}, \gamma_{2} \in \mathcal{M}(X)$ satisfying the mass balance condition

$$
\gamma_{1}(X)=\gamma_{2}(X)
$$

is the infimum

$$
\inf _{T \# \gamma_{1}=\gamma_{2}} \int_{X}|x-T(x)| d \gamma_{1}(x)
$$

attained among mappings $T$ which push $\gamma_{1}$ forward to $\gamma_{2}$ ? In the case that $\gamma_{1}$ and $\gamma_{2}$ represent the distributions for production and consumption of some commodity, the problem is then to decide which producer should supply each consumer to minimize the total transport cost.

In general, the Monge problem is ill-posed. To overcome the difficulties of the Monge problem, in 1942, L. V. Kantorovich ([10]) proposed to study a relaxed version of the Monge problem and, what is more relevant here, introduced a dual variational principle.

We will use the usual convention of denoting by $\pi_{i}: \mathbb{R}^{N} \times \mathbb{R}^{N} \rightarrow \mathbb{R}^{N}$ the projections, $\pi_{1}(x, y):=x$ and $\pi_{2}(x, y):=y$. Given a Radon measure $\mu$ in $X \times X$, its marginals are defined by $\operatorname{proj}_{x}(\mu):=\pi_{1} \# \mu$ and $\operatorname{proj}_{y}(\mu):=\pi_{2} \# \mu$.

The Monge-Kantorovich problem. Fix two measures $\gamma_{1}, \gamma_{2} \in \mathcal{M}(X)$ satisfying the mass balance condition (2.1). Let $\Pi\left(\gamma_{1}, \gamma_{2}\right)$ be the set of transport plans between $\gamma_{1}$ and $\gamma_{2}$, that is, the set of nonnegative Radon measures $\mu$ in $X \times X$ such that $\operatorname{proj}_{x}(\mu)=\gamma_{1}$ and $\operatorname{proj}_{y}(\mu)=\gamma_{2}$. The Monge-Kantorovich problem is to find a measure $\mu^{*} \in \Pi\left(\gamma_{1}, \gamma_{2}\right)$ which minimizes the cost functional

$$
\mathcal{K}(\mu):=\int_{X \times X}|x-y| d \mu(x, y),
$$

on the set $\Pi\left(\gamma_{1}, \gamma_{2}\right)$. A minimizer $\mu^{*}$ is called an optimal transport plan between $\gamma_{1}$ and $\gamma_{2}$.

Linearity makes the Monge-Kantorovich problem simpler than the original Monge problem; a continuity-compactness argument at least guarantees the existence of an optimal transport plan.

It is well known that linear minimization problems such as the Monge-Kantorovich problem admit dual formulations. In the context of optimal mass transportation, this was introduced by Kantorovich in 1942 ([10]). He established the following result.

Kantorovich duality. Fix two measures $\gamma_{1}, \gamma_{2} \in \mathcal{M}(X)$ satisfying the mass balance condition (2.1). For $(\varphi, \psi) \in L^{1}\left(d \gamma_{1}\right) \times L^{1}\left(d \gamma_{2}\right)$, define

$$
J(\varphi, \psi):=\int_{X} \varphi d \gamma_{1}+\int_{X} \psi d \gamma_{2},
$$


and let $\Phi$ be the set of all measurable functions $(\varphi, \psi) \in L^{1}\left(d \gamma_{1}\right) \times L^{1}\left(d \gamma_{2}\right)$ satisfying

$$
\varphi(x)+\psi(y) \leq|x-y| \quad \text { for } \gamma_{1} \times \gamma_{2}-\text { almost all }(x, y) \in X \times X .
$$

Then

$$
\inf _{\mu \in \Pi\left(\gamma_{1}, \gamma_{2}\right)} \mathcal{K}(\mu)=\sup _{(\varphi, \psi) \in \Phi} J(\varphi, \psi)
$$

The above result is true for more general cost functions than those corresponding to the Euclidean distance $|x-y|$. Now, for cost functions associated with lower semicontinuous distances there is a more precise result (see for instance Theorem 1.14 in [13]), which for the Euclidean distance can be written as follows.

Kantorovich-Rubinstein theorem. Let $\gamma_{1}, \gamma_{2} \in \mathcal{M}(X)$ be two measures satisfying the mass balance condition (2.1). Then,

$$
\min \left\{\mathcal{K}(\mu): \mu \in \Pi\left(\gamma_{1}, \gamma_{2}\right)\right\}=\sup \left\{\int_{X} u d\left(\gamma_{1}-\gamma_{2}\right): u \in K_{1}(X)\right\}
$$

where

$$
K_{1}(X):=\{u: X \rightarrow \mathbb{R}:|u(x)-u(y)| \leq|x-y| \quad \forall x, y \in X\}
$$

is the set of 1-Lipschitz functions in $X$.

The maximizers $u^{*}$ of the right-hand side of (2.2) are called Kantorovich (transport) potentials.

In the particular case where $\gamma_{1}=f_{+} \mathcal{L}^{N}$ and $\gamma_{2}=f_{-} \mathcal{L}^{N}$, for adequate Lebesgue integrable functions $f_{+}$and $f_{-}$, Evans and Gangbo in [7] find a Kantorovich potential as a limit, as $p \rightarrow \infty$, of solutions to the $p$-Laplace equation with Dirichlet boundary conditions in a sufficiently large ball $B(0, R)$ :

$$
\begin{cases}-\Delta_{p} u_{p}=f_{+}-f_{-} & \text {in } B(0, R) \\ u_{p}=0 & \text { on } \partial B(0, R) .\end{cases}
$$

Moreover, they characterize the Kantorovich potential by means of a PDE.

Evans-Gangbo theorem. Let $f_{+}, f_{-} \in L^{1}(\Omega)$ be two nonnegative Borel functions satisfying the mass balance condition $\int_{\Omega} f_{+}=\int_{\Omega} f_{-}$. Assume additionally that $f_{+}$and $f_{-}$are Lipschitz continuous functions with compact support such that $\operatorname{supp}\left(f_{+}\right) \cap \operatorname{supp}\left(f_{-}\right)=\emptyset$. Let $u_{p}$ be the solution of (2.3). Then the $u_{p}$ converge uniformly to $u^{*} \in K_{1}(\Omega)$ as $p \rightarrow \infty$. The limit $u^{*}$ verifies

$$
\int_{\Omega} u^{*}(x)\left(f_{+}(x)-f_{-}(x)\right) d x=\max \left\{\int_{\Omega} u(x)\left(f_{+}(x)-f_{-}(x)\right) d x: u \in K_{1}(\Omega)\right\},
$$

and moreover, there exists $0 \leq a \in L^{\infty}(\Omega)$ such that

$$
f_{+}-f_{-}=-\operatorname{div}\left(a \nabla u^{*}\right) \quad \text { in } \mathcal{D}^{\prime}(\Omega) .
$$

Furthermore, $\left|\nabla u^{*}\right|=1$ a.e. in the set $\{a>0\}$. 
The function $a$ that appears in the Evans-Gangbo theorem is the Lagrange multiplier corresponding to the constraint $\left|\nabla u^{*}\right| \leq 1$, and it is called the transport density. Moreover, what is very important from the point of view of mass transport is that Evans and Gangbo used this PDE to find a proof of the existence of an optimal transport map for the classical Monge problem, different to the first one given by Sudakov in 1979 by means of probabilistic methods ([11]; see also [2] and [4]).

\subsection{The mass transport problem with import/export taxes}

Assume (following [13]) that a businessman produces some product in some factories represented by $f_{+}$in $\Omega$ (note that $f_{+}$encodes the amount produced and its location). There are also some consumers of the product in $\Omega$ with a distribution given by $f_{-}$also defined in $\Omega$. The goal of the businessman is to transport all the mass (there is an infinite-cost penalty for not transporting the total mass) $f_{+}$to $f_{-}$ (to satisfy the consumers) or to the boundary (to export the product). In doing this, he pays the transport costs (given by the Euclidean distance) and when a unit of mass is left at a point $y \in \partial \Omega$ an additional cost given by $T_{e}(y)$, the export taxes. He also has the constraint of satisfying the demand of the consumers (there is also an infinite-cost penalty for not covering the demand), that is, he has to import product, if necessary, from the exterior (paying the transport costs plus the extra cost $T_{i}(x)$, the import taxes, for each unit of mass that enters $\Omega$ at the point $x \in \partial \Omega$ ). He has the freedom to choose to export or import mass provided he transports all the mass in $f_{+}$and covers all the mass of $f_{-}$, and of course the transport must satisfy the natural balance of masses. Observe also that, by assuming that $\Omega$ is convex, we guarantee that the boundary of $\Omega$ is not crossed when the mass is transported inside $\Omega$. This is the only point where we use the fact that $\Omega$ is convex.

His main goal is to minimize the total cost of this operation (distribution of production to satisfy consumers with export/import payment if convenient, minimizing the total cost that is given by the transport cost plus export/import taxes). In other words, the main goal is, given the set

$$
\mathcal{A}\left(f_{+}, f_{-}\right):=\left\{\begin{array}{r}
\mu \in \mathcal{M}^{+}(\bar{\Omega} \times \bar{\Omega}): \pi_{1} \# \mu\left\llcorner\Omega=f_{+} \mathcal{L}^{N}\llcorner\Omega\right. \\
\text { and } \pi_{2} \# \mu\left\llcorner\Omega=f_{-} \mathcal{L}^{N}\llcorner\Omega\right.
\end{array}\right\},
$$

to obtain

$$
\min \left\{\int_{\bar{\Omega} \times \bar{\Omega}}|x-y| d \mu+\int_{\partial \Omega} T_{i} d\left(\pi_{1} \# \mu\right)+\int_{\partial \Omega} T_{e} d\left(\pi_{2} \# \mu\right): \mu \in \mathcal{A}\left(f_{+}, f_{-}\right)\right\} .
$$

As we will see in the next section, this is the description of the mass transport problem involved in the above maximization problem obtained by the limiting procedure on (1.1) as $p \rightarrow \infty$.

To clarify this relation, let us give the following argument. A clever fellow proposes to the businessman to leave to him the planning and offers him the following deal: to pick up a unit of product at $x \in \Omega$ he will charge him $\varphi(x)$, and 
to pick it up at $x \in \partial \Omega, T_{i}(x)$ (paying the taxes the clever fellow); and to leave a unit of product at the consumer's location $y \in \Omega$ he will charge him $\psi(y)$, and for leaving it at $y \in \partial \Omega, T_{e}(y)$ (paying the taxes the clever fellow). Moreover, he proposes that he will do all this in such a way that

$$
\varphi(x)+\psi(y) \leq|x-y|
$$

and

$$
-T_{i} \leq \varphi \text { and }-T_{e} \leq \psi \quad \text { on } \partial \Omega
$$

In addition he guarantees some compensation (assuming negative payments if necessary; that is, $\varphi$ and $\psi$ are not necessarily nonnegative). Note that (2.4) is a natural requirement since, otherwise, the businessman could do the job by himself and that (2.5) is also natural since, otherwise, the fellow makes a gift to the businessman. Observe also that (2.4) and (2.5) imply

$$
-T_{i}(x)-T_{e}(y) \leq|x-y| \quad \forall x, y \in \partial \Omega
$$

which is a natural condition because it says that if one imports some mass from $x$ and exports it to $y$, he gets no benefit. This condition, for $x=y \in \partial \Omega$, says that

$$
T_{i}(x)+T_{e}(x) \geq 0
$$

i.e., at a given point the sum of exportation and importation taxes is nonnegative.

We now introduce the operator $J: C(\bar{\Omega}) \times C(\bar{\Omega}) \rightarrow \mathbb{R}$, defined by

$$
J(\varphi, \psi):=\int_{\Omega} \varphi(x) f_{+}(x) d x+\int_{\Omega} \psi(y) f_{-}(y) d y
$$

and let

$$
\mathcal{B}\left(T_{i}, T_{e}\right):=\left\{\begin{array}{r}
(\varphi, \psi) \in C(\bar{\Omega}) \times C(\bar{\Omega}): \varphi(x)+\psi(y) \leq|x-y| \\
-T_{i} \leq \varphi,-T_{e} \leq \psi \text { on } \partial \Omega
\end{array}\right\} .
$$

The aim of the fellow that helps the businessman is to obtain

$$
\sup \left\{J(\varphi, \psi):(\varphi, \psi) \in \mathcal{B}\left(T_{i}, T_{e}\right)\right\} \text {. }
$$

Now, given $(\varphi, \psi) \in \mathcal{B}\left(T_{i}, T_{e}\right)$ and $\mu \in \mathcal{A}\left(f_{+}, f_{-}\right)$we have

$$
\begin{aligned}
J(\varphi, \psi) & =\int_{\Omega} \varphi(x) f_{+}(x) d x+\int_{\Omega} \psi(y) f_{-}(y) d y \\
& =\int_{\bar{\Omega}} \varphi(x) d \pi_{1} \# \mu-\int_{\partial \Omega} \varphi d \pi_{1} \# \mu+\int_{\bar{\Omega}} \psi(y) d \pi_{2} \# \mu-\int_{\partial \Omega} \psi d \pi_{2} \# \mu \\
& \leq \int_{\bar{\Omega} \times \bar{\Omega}}|x-y| d \mu+\int_{\partial \Omega} T_{i} d\left(\pi_{1} \# \mu\right)+\int_{\partial \Omega} T_{e} d\left(\pi_{2} \# \mu\right) .
\end{aligned}
$$


Therefore,

$$
\begin{aligned}
& \sup \left\{J(\varphi, \psi):(\varphi, \psi) \in \mathcal{B}\left(T_{i}, T_{e}\right)\right\} \\
& \leq \inf \left\{\int_{\bar{\Omega} \times \bar{\Omega}}|x-y| d \mu+\int_{\partial \Omega} T_{i} d\left(\pi_{1} \# \mu\right)+\int_{\partial \Omega} T_{e} d\left(\pi_{2} \# \mu\right): \mu \in \mathcal{A}\left(f_{+}, f_{-}\right)\right\}
\end{aligned}
$$

This inequality will imply that the businessman accepts the offer. However, in fact, there is no gap between the two costs as we will see in the next duality result whose proof uses ideas from [13].

Theorem 2.1. Assume that $T_{i}$ and $T_{e}$ satisfy

$$
-T_{i}(x)-T_{e}(y)<|x-y| \quad \forall x, y \in \partial \Omega
$$

Then,

$$
\begin{aligned}
& \sup \left\{J(\varphi, \psi):(\varphi, \psi) \in \mathcal{B}\left(T_{i}, T_{e}\right)\right\} \\
& =\min \left\{\int_{\bar{\Omega} \times \bar{\Omega}}|x-y| d \mu+\int_{\partial \Omega} T_{i} d \pi_{1} \# \mu+\int_{\partial \Omega} T_{e} d \pi_{2} \# \mu: \mu \in \mathcal{A}\left(f_{+}, f_{-}\right)\right\} .
\end{aligned}
$$

Proof. By (2.8), there exist $\varphi_{0}, \psi_{0} \in C(\bar{\Omega}), \varphi_{0 \mid \partial \Omega} \leq T_{i}$ and $\psi_{0 \mid \partial \Omega} \leq T_{e}$, such that

$$
-\varphi_{0}(x)-\psi_{0}(y)<|x-y| \quad \forall x, y \in \Omega .
$$

In fact, let $L<1$ (close to 1 ) be such that

$$
-T_{i}(x)-T_{e}(y)<L|x-y| \quad \forall x, y \in \partial \Omega,
$$

and let

$$
\varphi_{0}(x)=\min _{y \in \partial \Omega}\left\{T_{i}(y)+L|x-y|\right\} .
$$

Taking $x=y \in \partial \Omega$ we obtain

$$
\varphi_{0}(x)=\min _{y \in \partial \Omega}\left\{T_{i}(y)+L|x-y|\right\} \leq T_{i}(x) .
$$

Now, we have

$$
-T_{i}(x)-L|x-y|<T_{e}(y) \quad \forall x, y \in \partial \Omega,
$$

and there exists a small $\varepsilon>0$ such that

$$
-T_{i}(x)-L|x-y|+\varepsilon<T_{e}(y) \quad \forall x, y \in \partial \Omega .
$$

Hence

$$
\psi_{0}(y)=-\min _{x \in \partial \Omega}\left(T_{i}(x)+L|x-y|-\varepsilon\right)<T_{e}(y) .
$$

Finally, we have,

$$
\begin{aligned}
\varphi_{0}(x)+\psi_{0}(y) & =\min _{z \in \partial \Omega}\left\{T_{i}(z)+L|x-z|\right\}-\min _{z \in \partial \Omega}\left(T_{i}(z)+L|z-y|\right)+\varepsilon \\
& \geq-L|x-y|+\varepsilon>-|x-y| .
\end{aligned}
$$


Now, the proof follows the ideas of the proof of the Kantorovich duality theorem given in [13]. Let us introduce operators

$$
\Theta, \Psi: C(\bar{\Omega} \times \bar{\Omega}) \rightarrow[0,+\infty] .
$$

The operator $\Theta$ is defined by

$$
\Theta(u):= \begin{cases}0 & \text { if } u(x, y) \geq-|x-y| \\ +\infty & \text { else. }\end{cases}
$$

Before defining the operator $\Psi$, let us define, for $u \in C(\bar{\Omega} \times \bar{\Omega})$,

$$
\mathcal{A}(u):=\left\{\begin{aligned}
(\varphi, \psi) \in C(\bar{\Omega}) \times C(\bar{\Omega}): u(x, y) & =\varphi(x)+\psi(y), \\
T_{i} & \geq \varphi, T_{e} \geq \psi \text { on } \partial \Omega
\end{aligned}\right\} .
$$

In the case $\mathcal{A}(u) \neq \emptyset$, we have that there exists

$$
\min _{(\varphi, \psi) \in \mathcal{A}(u)} \int_{\Omega} \varphi(x) f_{+}(x) d x+\int_{\Omega} \psi(y) f_{-}(y) d y .
$$

In fact, fix $(\tilde{\varphi}, \tilde{\psi}) \in \mathcal{A}(u)$. Then, for any $(\varphi, \psi) \in \mathcal{A}(u)$, we have

$$
\varphi(x)-\tilde{\varphi}(x)=\tilde{\psi}(y)-\psi(y)=\alpha \quad \forall x, y \in \bar{\Omega},
$$

consequently

$$
\begin{aligned}
\int_{\Omega} \varphi(x) f_{+}(x) d x+\int_{\Omega} \psi(y) f_{-}(y) d y= & \int_{\Omega} \tilde{\varphi}(x) f_{+}(x) d x+\int_{\Omega} \tilde{\psi}(y) f_{-}(y) d y \\
& +\alpha \int_{\Omega}\left(f_{+}(x)-f_{-}(x)\right) d x .
\end{aligned}
$$

Moreover,

$$
\alpha=\varphi(x)-\tilde{\varphi}(x) \leq T_{i}(x)-\tilde{\varphi}(x) \leq k_{1}
$$

and

$$
\alpha=\tilde{\psi}(y)-\psi(y) \geq \tilde{\psi}(y)-T_{e}(y) \geq-k_{2} .
$$

Therefore, given a sequence $\left\{\left(\varphi_{n}, \psi_{n}\right)\right\}$ minimizing (2.11), since

$$
\alpha_{n}=\varphi_{n}(x)-\tilde{\varphi}(x)=\tilde{\psi}(y)-\psi_{n}(y)
$$

with $-k_{2} \leq \alpha_{n} \leq k_{1}$, if we let

$$
\alpha:=\lim _{n \rightarrow \infty} \alpha_{n}
$$

and

$$
\varphi=\tilde{\varphi}+\alpha, \quad \psi=\tilde{\psi}-\alpha,
$$

we have that $(\varphi, \psi)$ is a minimizer of $(2.11)$. 
Having in mind (2.11), the operator $\Psi$ is defined by

$$
\Psi(u):= \begin{cases}\min _{(\varphi, \psi) \in \mathcal{A}(u)} \int_{\Omega} \varphi(x) f_{+}(x) d x+\int_{\Omega} \psi(y) f_{-}(y) d y & \text { if } \mathcal{A}(u) \neq \emptyset \\ +\infty & \text { if } \mathcal{A}(u)=\emptyset\end{cases}
$$

Clearly, $\Theta$ and $\Psi$ are convex functionals on $C(\bar{\Omega} \times \bar{\Omega})$. By $(2.10)$, for $u_{0}(x, y):=$ $\varphi_{0}(x)+\psi_{0}(y), \mathcal{A}\left(u_{0}\right) \neq \emptyset$ and $\Psi\left(u_{0}\right)<\infty$. Moreover, since $u_{0}(x, y)>-|x-y|$, we have $\Theta\left(u_{0}\right)=0$ and $\Theta$ is continuous at $u_{0}$. Then, we can apply the FenchelRockafellar duality theorem (see for instance Theorem 1.9. in [13]) to get

$$
\max _{\mu \in \mathcal{M}(\bar{\Omega} \times \bar{\Omega})}\left[-\Theta^{*}(-\mu)-\Psi^{*}(\mu)\right]=\inf _{u \in C(\bar{\Omega} \times \bar{\Omega})}[\Theta(u)+\Psi(u)],
$$

where $\Phi^{*}$ and $\Theta^{*}$ are the Legendre-Fenchel transforms of the operator $\Phi$ and $\Theta$, respectively.

Now, we compute both sides of (2.12). For the right-hand side we obtain,

$$
\begin{aligned}
\inf _{u \in C(\bar{\Omega} \times \bar{\Omega})}[\Theta(u)+\Psi(u)] & =\inf _{\substack{u \in C(\bar{\Omega} \times \bar{\Omega}): \\
u(x, y) \geq-|x-y| \\
\mathcal{A}(u) \neq \emptyset}} \Psi(u) \\
& =\inf _{\substack{\varphi, \psi \in C(\bar{\Omega}): \\
\varphi(x)+\psi(y) \geq-y \mid \\
T_{i} \geq \varphi, T_{e} \geq \psi \text { on } \partial \Omega}} \int_{\Omega} \varphi(x) f_{+}(x) d x+\int_{\Omega} \psi(y) f_{-}(y) d y,
\end{aligned}
$$

from which it follows that

$$
\inf _{u \in C(\bar{\Omega} \times \bar{\Omega})}[\Theta(u)+\Psi(u)]=-\sup \left\{J(\varphi, \psi):(\varphi, \psi) \in \mathcal{B}\left(T_{i}, T_{e}\right)\right\}
$$

For the left-hand side of (2.12) we first compute the Legendre-Fenchel transforms of the operators $\Theta$ and $\Psi$. For $\mu \in \mathcal{M}(\bar{\Omega} \times \bar{\Omega})$, we have

$$
\begin{aligned}
\Theta^{*}(-\mu) & =\sup _{u \in C(\bar{\Omega} \times \bar{\Omega})}\left(-\int_{\bar{\Omega} \times \bar{\Omega}} u(x, y) d \mu(x, y)-\Theta(u)\right) \\
& =\sup _{\substack{u \in \in(\bar{\Omega} \times \bar{\Omega}) \\
u(x, y) \geq-|x-y|}}-\int_{\bar{\Omega} \times \bar{\Omega}} u(x, y) d \mu(x, y) .
\end{aligned}
$$

Hence,

$$
\Theta^{*}(-\mu)= \begin{cases}\int_{\bar{\Omega} \times \Omega}|x-y| d \mu(x, y) & \text { if } \mu \in \mathcal{M}^{+}(\bar{\Omega} \times \bar{\Omega}) \\ +\infty & \text { otherwise. }\end{cases}
$$


On the other hand,

$$
\begin{aligned}
& \Psi^{*}(\mu) \\
& =\sup _{\substack{u \in C(\bar{\Omega} \times \bar{\Omega}) \\
\mathcal{A}(u) \neq \emptyset}}\left(\int_{\bar{\Omega} \times \bar{\Omega}} u(x, y) d \mu(x, y)-\min _{(\varphi, \psi) \in \mathcal{A}(u)} \int_{\Omega} \varphi(x) f_{+}(x) d x+\int_{\Omega} \psi(y) f_{-}(y) d y\right) \\
& =\sup _{\substack{\varphi, \psi \in C(\bar{\Omega}) \\
T_{i} \geq \varphi, T_{e} \geq \psi \\
\text { on } \partial \Omega}}\left(\int_{\bar{\Omega} \times \bar{\Omega}}(\varphi(x)+\psi(y)) d \mu(x, y)-\int_{\Omega} \varphi(x) f_{+}(x) d x-\int_{\Omega} \psi(y) f_{-}(y) d y\right) \\
& =\sup _{\substack{\varphi, \psi \in C(\bar{\Omega}) \\
T_{i} \geq \varphi, T_{e} \geq \psi \\
\text { on } \partial \Omega}}\left(\int_{\bar{\Omega}}\left(\varphi d \pi_{1} \# \mu+\psi d \pi_{1} \# \mu\right)-\int_{\Omega} \varphi(x) f_{+}(x) d x-\int_{\Omega} \psi(y) f_{-}(y) d y\right) .
\end{aligned}
$$

Hence,

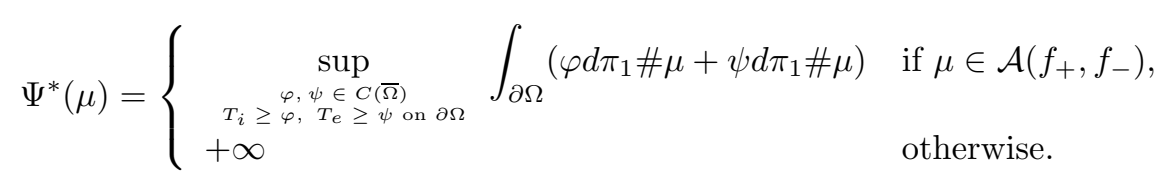

Therefore,

$$
\begin{aligned}
\max _{\mu \in \mathcal{M}(\bar{\Omega} \times \bar{\Omega})}\left[-\Theta^{*}(-\mu)-\Psi^{*}(\mu)\right. & =-\min _{\mu \in \mathcal{M}(\bar{\Omega} \times \bar{\Omega})}\left[\Theta^{*}(-\mu)+\Psi^{*}(\mu)\right] \\
& =-\min _{\substack{\mu \in \mathcal{M}^{+}(\bar{\Omega} \times \bar{\Omega}) \\
\mu \in \mathcal{A}\left(f_{+}, f_{-}\right)}} \int_{\bar{\Omega} \times \bar{\Omega}}|x-y| d \mu(x, y)+\int_{\partial \Omega} T_{i} d \pi_{1} \# \mu+\int_{\partial \Omega} T_{e} d \pi_{1} \# \mu .
\end{aligned}
$$

Then, from the above expression, (2.13) and (2.12), we get (2.9).

Remark 2.2. We remark that we can prove the above result for any lower semicontinuous cost function. Nevertheless, we have restricted ourselves to the Euclidean distance for the sake of clarity.

\section{Duality, Kantorovich potentials and optimal export/im- port masses}

In this section we prove the main results of this paper. As we have mentioned in the introduction, our approach is based on taking the limit, as $p$ goes to infinity, of least energy solutions of the $p$-Laplacian problem (1.2). This will give a complete proof of the duality theorem (note that this proof is different from the previous one). Moreover, this approach also gives more detailed information for the transport problem under consideration. It provides an explicit approximation of the Kantorovich potential $u_{\infty}$ and describes the required import/export masses on the boundary. In addition, we obtain transport plans constructed via transport maps.

First, we prove that we can take the limit of the functions $u_{p}$ solving the minimization problem (1.1) as $p \rightarrow \infty$. 
Theorem 3.1. Assume that $g_{1}$ and $g_{2}$ satisfy (1.3). Then, there is a sequence $p_{i} \rightarrow+\infty$ such that $u_{p_{i}} \rightarrow u_{\infty}$ uniformly as $i \rightarrow \infty$, and $u_{\infty}$ is a maximizer of the variational problem

$$
\max \left\{\int_{\Omega} w(x) f(x) d x: w \in W_{g_{1}, g_{2}}^{1, \infty}(\Omega),\|\nabla w\|_{L^{\infty}(\Omega)} \leq 1\right\} .
$$

Proof. Assume $p>N$. We first show that there exists a function $w \in W_{g_{1}, g_{2}}^{1, \infty}(\Omega)$ with $\|\nabla w\|_{L^{\infty}(\Omega)} \leq 1$. Define

$$
w(x)=\max _{y \in \partial \Omega}\left\{g_{1}(y)-|x-y|\right\} .
$$

We have that $|w(x)-w(y)| \leq|x-y|$ and (taking $x=y \in \partial \Omega$ in the definition of $w$ )

$$
w(x) \geq g_{1}(x) \text { for } x \in \partial \Omega .
$$

Moreover, as (1.3) holds, we have

$$
g_{1}(y)-|x-y| \leq g_{2}(x) \quad \forall x, y \in \partial \Omega,
$$

and hence we obtain

$$
w(x)=\max _{y \in \partial \Omega}\left\{g_{1}(y)-|x-y|\right\} \leq g_{2}(x) \quad \forall x \in \partial \Omega .
$$

Therefore, $w \in W_{g_{1}, g_{2}}^{1, \infty}(\Omega)$ with $\|\nabla w\|_{L^{\infty}(\Omega)} \leq 1$. Moreover, for any such $w$,

$$
-\int_{\Omega} f u_{p} \leq \frac{1}{p} \int_{\Omega}\left|\nabla u_{p}\right|^{p}-\int_{\Omega} f u_{p} \leq \frac{1}{p} \int_{\Omega}|\nabla w|^{p}-\int_{\Omega} f w \leq \frac{|\Omega|}{p}-\int_{\Omega} f w .
$$

As a consequence of Theorem 2.E in [12], there holds Morrey's inequality

$$
\|u\|_{L^{\infty}(\Omega)} \leq C_{\Omega}\|\nabla u\|_{L^{p}(\Omega)} \quad \text { for any } u \in W_{0}^{1, p}(\Omega), p>N,
$$

with a constant $C_{\Omega}$ not depending on $p$. Since $\left(u_{p}-\max _{\partial \Omega} g_{2}\right)^{+},\left(u_{p}-\min _{\partial \Omega} g_{1}\right)^{-} \in$ $W_{0}^{1, p}(\Omega)$, applying inequality $(3.2)$, we get

$$
\left\|u_{p}^{+}\right\|_{L^{\infty}(\Omega)} \leq C_{\Omega}\left\|\nabla u_{p}\right\|_{L^{p}(\Omega)}+\left|\max _{\partial \Omega} g_{2}\right|
$$

and

$$
\left\|u_{p}^{-}\right\|_{L^{\infty}(\Omega)} \leq C_{\Omega}\left\|\nabla u_{p}\right\|_{L^{p}(\Omega)}+\left|\min _{\partial \Omega} g_{1}\right|
$$

Hence, we have

$$
\left\|u_{p}\right\|_{L^{\infty}(\Omega)} \leq 2 C_{\Omega}\left\|\nabla u_{p}\right\|_{L^{p}(\Omega)}+\left\|g_{1}\right\|_{L^{\infty}(\partial \Omega)}+\left\|g_{2}\right\|_{L^{\infty}(\partial \Omega)}
$$

That is,

$$
\left\|u_{p}\right\|_{L^{\infty}(\Omega)} \leq C_{1}\left\|\nabla u_{p}\right\|_{L^{p}(\Omega)}+C_{2},
$$


where the constants $C_{i}$ are independent of $p$. Moreover, from (3.1), using Hölder's inequality and having in mind (3.3), we get

$$
\frac{1}{p} \int_{\Omega}\left|\nabla u_{p}\right|^{p} \leq C_{3}\left(\left\|u_{p}\right\|_{L^{p}(\Omega)}+1\right) \leq C_{4}\left(\left\|\nabla u_{p}\right\|_{L^{p}(\Omega)}+1\right),
$$

from which it follows that

$$
\left\|\nabla u_{p}\right\|_{L^{p}(\Omega)}^{p-1} \leq p C_{5} \quad \forall p>N
$$

with all the constants $C_{i}$ independent of $p$. From (3.3) and (3.4), we obtain that the $W^{1, p}(\Omega)$-norms of the $u_{p}$ are uniformly bounded for $p>N$ in $\mathbb{R}$. As a simple consequence, we have that

$$
\left|u_{p}(x)-u_{p}(y)\right| \leq C_{6}|x-y|^{1-N / p},
$$

with $C_{6}$ not depending on $p$. Then, by the Morrey-Sobolev embedding theorem and the Arzelà-Ascoli compactness criterion we can extract a sequence $p_{i} \rightarrow \infty$ such that

$$
u_{p_{i}} \rightrightarrows u_{\infty} \quad \text { uniformly in } \bar{\Omega} .
$$

Moreover, by (3.4), we obtain that

$$
\left\|\nabla u_{\infty}\right\|_{\infty} \leq 1
$$

Finally, passing to the limit in (3.1), we get

$$
\int_{\Omega} u_{\infty}(x) f(x) d x=\max \left\{\int_{\Omega} w(x) f(x) d x: w \in W_{g_{1}, g_{2}}^{1, \infty}(\Omega),\|\nabla w\|_{L^{\infty}(\Omega)} \leq 1\right\},
$$

whic is what we wanted to prove.

Now we present the general duality result that proves that the businessman and his business partner pay the same total cost under the natural condition (2.6) (note that the strict inequality is not necessary), giving a positive answer to the main problem stated in the introduction. In this result the role of $T_{i}$ is played by $-g_{1}$, and the role of $T_{e}$, by $g_{2}((2.6)$ is, then, equivalent to $(1.3))$.

Theorem 3.2. If $g_{1}$ and $g_{2}$ satisfy (1.3) then

$$
\begin{aligned}
& \int_{\Omega} u_{\infty}(x)\left(f_{+}(x)-f_{-}(x)\right) d x=\sup \left\{J(\varphi, \psi):(\varphi, \psi) \in \mathcal{B}\left(-g_{1}, g_{2}\right)\right\} \\
& =\min \left\{\int_{\bar{\Omega} \times \bar{\Omega}}|x-y| d \mu-\int_{\partial \Omega} g_{1} d \pi_{1} \# \mu+\int_{\partial \Omega} g_{2} d \pi_{2} \# \mu: \mu \in \mathcal{A}\left(f_{+}, f_{-}\right)\right\},
\end{aligned}
$$

where $u_{\infty}$ is the maximizer given in Theorem 3.1.

Before proving this result we make the following observation. 
Remark 3.3. Fix $\mu \in \mathcal{A}\left(f_{+}, f_{-}\right)$, a minimizer of (3.5). If $\mu_{i}:=\pi_{i} \# \mu, i=1,2$, by the Kantorovich-Rubinstein theorem, we have

$$
\begin{aligned}
& \min \left\{\int_{\bar{\Omega} \times \bar{\Omega}}|x-y| d \nu: \nu\right.\left.\in \Pi\left(\mu_{1}, \mu_{2}\right)\right\} \\
&=\max \left\{\int_{\bar{\Omega}} u d\left(\mu_{1}-\mu_{2}\right): u \in K_{1}(\bar{\Omega})\right\} .
\end{aligned}
$$

We show that $\mu$ is an optimal transport plan for (3.6). Indeed, if $\nu_{\mu} \in \Pi\left(\mu_{1}, \mu_{2}\right)$ is an optimal transport plan for (3.6), then, as $\mu \in \Pi\left(\mu_{1}, \mu_{2}\right)$,

$$
\int_{\bar{\Omega} \times \bar{\Omega}}|x-y| d \nu_{\mu} \leq \int_{\bar{\Omega} \times \bar{\Omega}}|x-y| d \mu .
$$

Now, since

$$
\int_{\partial \Omega} g_{1} d \pi_{1} \# \nu_{\mu}-\int_{\partial \Omega} g_{2} d \pi_{2} \# \nu_{\mu}=\int_{\partial \Omega} g_{1} d \mu_{1}-\int_{\partial \Omega} g_{2} d \mu_{2},
$$

we have

$$
\begin{aligned}
\int_{\bar{\Omega} \times \bar{\Omega}}|x-y| d \nu_{\mu} & -\int_{\partial \Omega} g_{1} d \pi_{1} \# \nu_{\mu}+\int_{\partial \Omega} g_{2} d \pi_{2} \# \nu_{\mu} \\
& \leq \int_{\bar{\Omega} \times \bar{\Omega}}|x-y| d \mu-\int_{\partial \Omega} g_{1} d \mu_{1}+\int_{\partial \Omega} g_{2} d \mu_{2}
\end{aligned}
$$

On the other hand, since $\nu_{\mu} \in \mathcal{A}\left(f_{+}, f_{-}\right)$,

$$
\begin{aligned}
\int_{\bar{\Omega} \times \bar{\Omega}}|x-y| d \mu & -\int_{\partial \Omega} g_{1} d \mu_{1}+\int_{\partial \Omega} g_{2} d \mu_{2} \\
& \leq \int_{\bar{\Omega} \times \bar{\Omega}}|x-y| d \nu_{\mu}-\int_{\partial \Omega} g_{1} d \pi_{1} \# \nu_{\mu}+\int_{\partial \Omega} g_{2} d \pi_{2} \# \nu_{\mu} .
\end{aligned}
$$

Therefore, the above inequality is an equality and then

$$
\int_{\bar{\Omega} \times \bar{\Omega}}|x-y| d \mu=\int_{\bar{\Omega} \times \bar{\Omega}}|x-y| d \nu_{\mu},
$$

and consequently $\mu$ is an optimal transport plan for (3.6).

Let $u^{*}$ be a Kantorovich potential in (3.6). Then

$$
\int_{\bar{\Omega} \times \bar{\Omega}}|x-y| d \mu=\int_{\bar{\Omega}} u^{*} d\left(\mu_{1}-\mu_{2}\right) .
$$

Hence,

$$
\begin{aligned}
\int_{\Omega} u_{\infty}\left(f_{+}-f_{-}\right) d x & =\int_{\bar{\Omega} \times \bar{\Omega}}|x-y| d \mu-\int_{\partial \Omega} g_{1} d \mu_{1}+\int_{\partial \Omega} g_{2} d \mu_{2} \\
& =\int_{\bar{\Omega}} u^{*} d\left(\mu_{1}-\mu_{2}\right)-\int_{\partial \Omega} g_{1} d \mu_{1}+\int_{\partial \Omega} g_{2} d \mu_{2},
\end{aligned}
$$


and, then,

$\int_{\bar{\Omega}} u_{\infty} d\left(\mu_{1}-\mu_{2}\right) \geq \int_{\Omega} u_{\infty}\left(f_{+}-f_{-}\right) d x+\int_{\partial \Omega} g_{1} d \mu_{1}-\int_{\partial \Omega} g_{2} \mu_{2}=\int_{\bar{\Omega}} u^{*} d\left(\mu_{1}-\mu_{2}\right)$.

That is, $u_{\infty}$ is also a Kantorovich potential for (3.6). From this last expression we also deduce that

$$
u_{\infty}=g_{i} \quad \text { on } \operatorname{supp}\left(\mu_{i}\llcorner\partial \Omega), i=1,2 .\right.
$$

We point out that there is an important difference between the problem we are studying and the classical transport problem; there are masses, the ones that appear on the boundary, that are unknown variables. We will see in the next result that by taking the limit of $u_{p}$, minimizers of (1.1), we obtain, not only the potential $u_{\infty}$, but also these masses. This result also proves Theorem 3.2. This is an alternative proof of Theorem 2.1 (note that in the previous proof the Kantorovich potentials were not used). Note that we first assume the more restrictive condition (3.7), that is, a strict inequality in (1.3), and then we obtain the result, assuming (1.3), by an approximation argument (see below).

Theorem 3.4. Assume that $g_{1}$ and $g_{2}$ verifies

$$
g_{1}(x)-g_{2}(y)<|x-y| \quad \forall x, y \in \partial \Omega .
$$

(1) Let $u_{p}$ be a minimizer of the problem (1.1), and set $\mathcal{X}_{p}:=\left|D u_{p}\right|^{p-2} D u_{p}$. The distribution $\mathcal{X}_{p} \cdot \eta$ defined by

$$
\left\langle\mathcal{X}_{p} \cdot \eta, \varphi\right\rangle:=\int_{\Omega} \mathcal{X}_{p} \cdot \nabla \varphi-\int_{\Omega} f \varphi \quad \text { for } \varphi \in C_{0}^{\infty}\left(\mathbb{R}^{N}\right),
$$

is a Radon measure supported on $\partial \Omega$.

(2) There exist Radon measures $\mathcal{X}$ in $\Omega$ and $\mathcal{V}$ in $\partial \Omega$, and a sequence $p_{i} \rightarrow+\infty$, such that

$$
\begin{gathered}
\mathcal{X}_{p_{i}} \rightarrow \mathcal{X} \quad \text { weakly }^{*} \text { in the sense of measures in } \Omega, \\
\mathcal{X}_{p_{i}} \cdot \eta \rightarrow \mathcal{V} \quad \text { weakly }^{*} \text { in the sense of measures in } \partial \Omega ; \\
\int_{\Omega} \nabla \varphi d \mathcal{X}=\int_{\Omega} f \varphi d x+\int_{\partial \Omega} \varphi d \mathcal{V} \quad \forall \varphi \in C^{1}(\bar{\Omega}) .
\end{gathered}
$$

(3) Let $u_{\infty}$ be as stated in Theorem 3.1. Then, $u_{\infty}$ is a Kantorovich potential for the classical transport problem for the measures $f_{+} \mathcal{L}^{N}\left\llcorner\Omega+\mathcal{V}^{+}\right.$and $f_{-} \mathcal{L}^{N}\left\llcorner\Omega+\mathcal{V}^{-}\right.$.

Proof. We begin by proving (1). Remember that we are considering $p>N$. Let $u_{p}$ be a minimizer of the problem (1.1), which is a solution of the obstacle problem (1.2), and let $\mathcal{X}_{p}=\left|D u_{p}\right|^{p-2} D u_{p}$. Then, we know that

$$
-\operatorname{div}\left(\mathcal{X}_{p}\right)=f \quad \text { in the sense of distributions in } \Omega .
$$


This implies that the $\mathcal{X}_{p} \cdot \eta$ defined in (3.8) is a distribution supported on $\partial \Omega$. We show that, in fact,

$$
\operatorname{supp}\left(\mathcal{X}_{p} \cdot \eta\right) \subset\left\{x \in \partial \Omega: u_{p}(x)=g_{1}(x)\right\} \cup\left\{x \in \partial \Omega: u_{p}(x)=g_{2}(x)\right\} .
$$

Let $\varphi$ be a smooth function such that

$$
\operatorname{supp}(\varphi) \cap\left(\left\{x \in \partial \Omega: u_{p}(x)=g_{1}(x)\right\} \cup\left\{x \in \partial \Omega: u_{p}(x)=g_{2}(x)\right\}\right)=\emptyset .
$$

Then, there exists $\delta>0$ such that $u_{p}+t \varphi \in W_{g_{1}, g_{2}}^{1, p}(\Omega)$ for all $|t|<\delta$. Hence, since $u_{p}$ is a minimizer of the problem (1.1), we have

$$
\int_{\Omega} f\left(u_{p}+t \varphi\right) d x-\int_{\Omega} f u_{p} d x \leq \int_{\Omega} \frac{\left|\nabla u_{p}+t \nabla \varphi\right|^{p}}{p} d x-\int_{\Omega} \frac{\left|\nabla u_{p}\right|^{p}}{p} d x .
$$

Dividing by $t$ and taking the limit as $t \rightarrow 0$, we get

$$
\int_{\Omega} \mathcal{X}_{p} \cdot \nabla \varphi d x=\int_{\Omega} f \varphi d x
$$

from which it follows, having in mind (3.10), that

$$
\left\langle\mathcal{X}_{p} \cdot \eta, \varphi\right\rangle=0
$$

Consequently (3.11) holds.

On the other hand, if $\varphi$ is a positive smooth function whose support does not touch $\left\{x \in \partial \Omega: u_{p}(x)=g_{2}(x)\right\}$ (which is separated from $\left\{x \in \partial \Omega: u_{p}(x)=g_{1}(x)\right\}$ by the continuity of $u_{p}$ and the strict inequality in (3.7)) then there exists $\delta>0$ such that $u_{p}+t \varphi \in W_{g_{1}, g_{2}}^{1, p}(\Omega)$ for all $0 \leq t<\delta$. Working as above we get

$$
\left\langle\mathcal{X}_{p} \cdot \eta, \varphi\right\rangle \geq 0
$$

And similarly, if $\varphi$ is a positive smooth function whose support does not touch $\left\{x \in \partial \Omega: u_{p}(x)=g_{1}(x)\right\}$,

$$
\left\langle\mathcal{X}_{p} \cdot \eta, \varphi\right\rangle \leq 0
$$

Consequently, $\mathcal{X}_{p} \cdot \eta$ is a Radon measure. The proof of this fact follows by writing $\mathcal{X}_{p} \cdot \eta=T_{1}+T_{2}$ with $\left\langle T_{i}, \varphi\right\rangle=\left\langle\mathcal{X}_{p} \cdot \eta, \varphi \varphi_{i}\right\rangle$ with $\varphi_{i} \in \mathcal{C}_{0}^{\infty}\left(\mathbb{R}^{N}\right)$ such that

$$
\varphi_{1}(x)= \begin{cases}1, & x \in \partial \Omega, u_{p}(x)=g_{1}(x) \\ 0, & x \in \partial \Omega, u_{p}(x)=g_{2}(x)\end{cases}
$$

and

$$
\varphi_{2}(x)= \begin{cases}1, & x \in \partial \Omega, u_{p}(x)=g_{2}(x), \\ 0, & x \in \partial \Omega, u_{p}(x)=g_{1}(x)\end{cases}
$$

and noticing that the above arguments show that $T_{1}$ and $-T_{2}$ are nonnegative distributions and so Radon measures. Moreover,

$$
\operatorname{supp}\left(\left(\mathcal{X}_{p} \cdot \eta\right)^{+}\right) \subset\left\{x \in \partial \Omega: u_{p}(x)=g_{1}(x)\right\},
$$


and

$$
\operatorname{supp}\left(\left(\mathcal{X}_{p} \cdot \eta\right)^{-}\right) \subset\left\{x \in \partial \Omega: u_{p}(x)=g_{2}(x)\right\} .
$$

In addition, we have that (3.8) is satisfied for test functions $\varphi \in W^{1, p}(\Omega)$ and we can rewrite it as

$$
\int_{\Omega} \mathcal{X}_{p} \cdot \nabla \varphi=\int_{\Omega} f \varphi+\int_{\partial \Omega} \varphi d\left(\mathcal{X}_{p} \cdot \eta\right) .
$$

Proof of (2). Using (3.7), there is $0<L<1$ such that

$$
g_{1}(x)-g_{2}(y)<L|x-y| \quad \forall x, y \in \partial \Omega .
$$

Therefore, if we define

$$
w(x):=\inf _{y \in \partial \Omega}\left(g_{2}(y)+L|x-y|\right),
$$

we have that $w$ is an $L$-Lipschitz function in $\bar{\Omega}$ satisfying

$$
g_{1}(x)<w(x) \leq g_{2}(x) \quad \forall x \in \partial \Omega
$$

By (3.14), (3.11), (3.12), and (3.13), we have

$$
\begin{aligned}
\int_{\Omega}\left(u_{p}-w\right) f= & \int_{\Omega} \mathcal{X}_{p} \cdot \nabla\left(u_{p}-w\right)-\int_{\partial \Omega}\left(u_{p}-w\right) d\left(\mathcal{X}_{p} \cdot \eta\right) \\
= & \int_{\Omega} \mathcal{X}_{p} \cdot \nabla\left(u_{p}-w\right)-\int_{\left\{g_{1}=u_{p}\right\}}\left(g_{1}-w\right) d\left(\mathcal{X}_{p} \cdot \eta\right)^{+} \\
& +\int_{\left\{g_{2}=u_{p}\right\}}\left(g_{2}-w\right) d\left(\mathcal{X}_{p} \cdot \eta\right)^{-} .
\end{aligned}
$$

Then, since $g_{1}-w \leq-c$, with $c>0$, and $g_{2}-w \geq 0$, by Hölder's and Young's inequalities, it follows that

$$
\begin{aligned}
\int_{\Omega}\left|\nabla u_{p}\right|^{p} & +c \int_{\partial \Omega} d\left(\mathcal{X}_{p} \cdot \eta\right)^{+} \leq \int_{\Omega}\left(u_{p}-w\right) f+\int_{\Omega} \mathcal{X}_{p} \cdot \nabla w \\
& \leq C+\left(\int_{\Omega}\left|\nabla u_{p}\right|^{p}\right)^{1 / p^{\prime}} L|\Omega|^{1 / p^{\prime}} \leq C+\frac{L}{p^{\prime}} \int_{\Omega}\left|\nabla u_{p}\right|^{p}+\frac{1}{p}|\Omega| .
\end{aligned}
$$

Hence,

$$
\left(1-\frac{L^{p^{\prime}}}{p^{\prime}}\right) \int_{\Omega}\left|\nabla u_{p}\right|^{p}+c \int_{\partial \Omega} d\left(\mathcal{X}_{p} \cdot \eta\right)^{+} \leq C+\frac{1}{p}|\Omega| .
$$

Therefore, since $0<L<1$ and $c>0$, we obtain that there exist positive constants $A_{1}$ and $A_{2}$, such that

$$
\int_{\Omega}\left|\nabla u_{p}\right|^{p} \leq A_{1}, \quad \forall p \geq N+1,
$$


and

$$
\int_{\partial \Omega} d\left(\mathcal{X}_{p} \cdot \eta\right)^{+} \leq A_{2}, \quad \forall p \geq N+1
$$

Moreover, working similarly, replacing the function $w$ by the function

$$
\tilde{w}(x)=\sup _{y \in \partial \Omega}\left(g_{1}(y)-L|x-y|\right)
$$

we get

$$
\int_{\partial \Omega} d\left(\mathcal{X}_{p} \cdot \eta\right)^{-} \leq A_{3}, \quad \forall p \geq N+1 .
$$

As consequence of (3.15), we have that

$$
\text { the measures } \mathcal{X}_{p} \mathcal{L}^{N}\llcorner\Omega \text { are equibounded in } \Omega \text {, }
$$

and from (3.16) and (3.17), we have that

$$
\text { the measures } \mathcal{X}_{p} \cdot \eta \mathcal{H}^{N-1}\llcorner\partial \Omega \text { are equibounded on } \partial \Omega \text {. }
$$

From (3.18) and (3.19), there exists a sequence $p_{i} \rightarrow \infty$, which we consider as subsequence of that in Theorem 3.1 and denote in the same way, and there exist Radon measures $\mathcal{X}$ in $\Omega$ and $\mathcal{V}$ in $\partial \Omega$ such that

$$
\mathcal{X}_{p_{i}} \rightarrow \mathcal{X} \quad \text { weakly* }^{*} \text { as measures in } \Omega
$$

and

$$
\mathcal{X}_{p_{i}} \cdot \eta \rightarrow \mathcal{V} \quad \text { weakly }^{*} \text { as measures on } \partial \Omega \text {. }
$$

Moreover, we have that (3.9) holds true. That is, formally,

$$
\begin{cases}-\operatorname{div}(\mathcal{X})=f & \text { in } \Omega \\ \mathcal{X} \cdot \eta=\mathcal{V} & \text { on } \partial \Omega\end{cases}
$$

Proof of (3). For the above sequence $\left\{p_{i}\right\}$, Theorem 3.1 states

$$
u_{p_{i}} \rightrightarrows u_{\infty} \quad \text { uniformly in } \bar{\Omega}, \quad \text { with }\left\|\nabla u_{\infty}\right\|_{\infty} \leq 1 .
$$

Set $\varphi=u_{\infty}$ in (3.14) with $p=p_{i}$. Then taking the limit as $i \rightarrow \infty$ and having in mind (3.21), we get

$$
\lim _{i \rightarrow \infty} \int_{\Omega} \mathcal{X}_{p_{i}} \cdot \nabla u_{\infty}=\int_{\Omega} f u_{\infty}+\int_{\partial \Omega} u_{\infty} d \mathcal{V}
$$

Let $v_{\epsilon}$ be smooth functions converging uniformly to $u_{\infty}$ as $\epsilon \searrow 0$ and satisfying $\left\|\nabla v_{\epsilon}\right\|_{\infty} \leq 1$. By (3.14), we have

$$
\int_{\Omega} \mathcal{X}_{p_{i}} \cdot \nabla u_{\infty}=\int_{\Omega} f\left(u_{\infty}-v_{\epsilon}\right)+\int_{\partial \Omega}\left(u_{\infty}-v_{\epsilon}\right) d\left(\mathcal{X}_{p_{i}} \cdot \eta\right)+\int_{\Omega} \nabla v_{\epsilon} d\left(\mathcal{X}_{p_{i}} \cdot \eta\right) .
$$


Then, by (3.20), (3.21), and (3.22), taking the limit in the above equality as $i \rightarrow \infty$, we obtain

$$
\int_{\Omega} f u_{\infty}+\int_{\partial \Omega} u_{\infty} d \mathcal{V}=\int_{\Omega} f\left(u_{\infty}-v_{\epsilon}\right)+\int_{\partial \Omega}\left(u_{\infty}-v_{\epsilon}\right) d \mathcal{V}+\int_{\Omega} \nabla v_{\epsilon} d \mathcal{X}
$$

Now we are going to show that, as $\epsilon \searrow 0$,

(3.24) $\nabla v_{\epsilon} \quad$ converges in $L^{2}(|\mathcal{X}|)$ to the Radon-Nikodym derivative $\frac{\mathcal{X}}{|\mathcal{X}|}$.

To do so we use the technique used in Theorem 5.2 of [2]. We first notice that the functional $\Psi:\left[C\left(\bar{\Omega}, \mathbb{R}^{N}\right)\right]^{*} \rightarrow \mathbb{R}$ defined by

$$
\Psi(\nu):=\int_{\bar{\Omega}}\left|\frac{\nu}{|\nu|}-w\right|^{2} d|\nu|
$$

is lower semicontinuous with respect to weak convergence of measures for any $w \in C\left(\bar{\Omega}, \mathbb{R}^{N}\right)$. Next, we observe that

$$
\lim _{\epsilon \rightarrow 0^{+}} \limsup _{i \rightarrow \infty} \int_{\Omega}\left|\frac{\mathcal{X}_{p_{i}}}{\left|\mathcal{X}_{p_{i}}\right|}-\nabla v_{\epsilon}\right|^{2} d\left|\mathcal{X}_{p_{i}}\right|=0
$$

where the $v_{\epsilon}$ are smooth functions converging uniformly to $u_{\infty}$ with $\left\|\nabla v_{\epsilon}\right\|_{\infty} \leq 1$. Indeed,

$$
\begin{aligned}
\int_{\Omega} \mid \frac{\mathcal{X}_{p_{i}}}{\left|\mathcal{X}_{p_{i}}\right|} & -\left.\nabla v_{\epsilon}\right|^{2} d\left|\mathcal{X}_{p_{i}}\right| \leq 2 \int_{\Omega}\left|\nabla u_{p_{i}}\right|^{p_{i}-1}\left(1-\frac{\nabla v_{\epsilon} \cdot \nabla u_{p_{i}}}{\left|\nabla u_{p_{i}}\right|}\right) d x \\
& \leq 2 \int_{\Omega}\left|\nabla u_{p_{i}}\right|^{p_{i}-2}\left(\left|\nabla u_{p_{i}}\right|^{2}-\nabla v_{\epsilon} \cdot \nabla u_{p_{i}}\right) d x+\omega_{p_{i}} \\
& =2 \int_{\Omega} f\left(u_{p_{i}}-v_{\epsilon}\right) d x+\int_{\partial \Omega}\left(u_{p_{i}}-v_{\epsilon}\right) d \mathcal{X}_{p_{i}} \cdot \eta+\omega_{p_{i}}
\end{aligned}
$$

where $\omega_{p_{i}}:=\sup _{t \geq 0} t^{p_{i}-1}-t^{p_{i}}$ tends to 0 as $i \rightarrow \infty$. Then, having in mind (3.21) and the uniform convergence of $u_{p_{i}}$ and $v_{\epsilon}$ to $u_{\infty}$, we obtain (3.25). Now, from (3.25), taking into account the lower semicontinuity of $\Psi$ and passing to the limit as $i \rightarrow \infty$, we obtain

$$
\lim _{\epsilon \rightarrow 0^{+}} \int_{\Omega}\left|\frac{\mathcal{X}}{|\mathcal{X}|}-\nabla v_{\epsilon}\right|^{2} d|\mathcal{X}|=0
$$

Consequently, (3.24) holds true.

Now, having in mind (3.24), if we take the limit in (3.23) as $\epsilon \searrow 0$, we get

$$
\int_{\Omega} f u_{\infty}+\int_{\partial \Omega} u_{\infty} d \mathcal{V}=\lim _{\epsilon \downarrow 0} \int_{\Omega} \nabla v_{\epsilon} \cdot \frac{\mathcal{X}}{|\mathcal{X}|} d|\mathcal{X}|=\int_{\Omega} d|\mathcal{X}|
$$

Given a function $\varphi \in C^{1}(\bar{\Omega})$ with $\|\nabla \varphi\|_{\infty} \leq 1$, by (3.9) and (3.26), we have

$$
\begin{aligned}
\int_{\Omega} u_{\infty} f d x+\int_{\partial \Omega} u_{\infty} d \mathcal{V} & =\int_{\Omega} d|\mathcal{X}| \geq \int_{\Omega} \frac{\mathcal{X}}{|\mathcal{X}|} \cdot \nabla \varphi d|\mathcal{X}| \\
& =\int_{\Omega} \nabla \varphi d \mathcal{X}=\int_{\Omega} \varphi f d x+\int_{\partial \Omega} \varphi d \mathcal{V}
\end{aligned}
$$


Then, by approximation, given a Lipschitz continuous function $w$ with $\|\nabla w\|_{\infty} \leq 1$, we obtain

$$
\int_{\Omega} u_{\infty} f d x+\int_{\partial \Omega} u_{\infty} d \mathcal{V} \geq \int_{\Omega} w f d x+\int_{\partial \Omega} w d \mathcal{V}
$$

Therefore $u_{\infty}$ is a Kantorovich potential for the classical transport problem associated to the measures $f_{+} \mathcal{L}^{N} L \Omega+\mathcal{V}^{+}$and $f_{-} \mathcal{L}^{N} L \Omega+\mathcal{V}^{-}$. Observe that the total masses of both measures are the same.

This provides a proof of Theorem 3.2, and moreover we have that $\mathcal{V}^{+}$and $\mathcal{V}^{-}$ are import and export masses in our original problem. In fact, they correspond to the import/export measures once we consider the suitable associated transport plan.

Proof of Theorem 3.2. It is enough to show that

$$
\begin{aligned}
& \int_{\Omega} u_{\infty}(x)\left(f_{+}(x)-f_{-}(x)\right) d x \\
& \quad=\min \left\{\int_{\bar{\Omega} \times \bar{\Omega}}|x-y| d \mu-\int_{\partial \Omega} g_{1} d \pi_{1} \# \mu+\int_{\partial \Omega} g_{2} d \pi_{2} \# \mu: \mu \in \mathcal{A}\left(f_{+}, f_{-}\right)\right\} .
\end{aligned}
$$

Let us first assume that

$$
g_{1}(x)-g_{2}(y)<|x-y| \quad \forall x, y \in \partial \Omega,
$$

and take $\tilde{f}_{1}:=f_{+} \mathcal{L}^{N}\left\llcorner\Omega+\mathcal{V}^{+}\right.$and $\tilde{f}_{2}:=f_{-} \mathcal{L}^{N}\left\llcorner\Omega+\mathcal{V}^{-}, \mathcal{V}\right.$ being the measure found in Theorem 3.4. We have that

$$
\int_{\bar{\Omega}} u_{\infty} d\left(\tilde{f}_{1}-\tilde{f}_{2}\right)=\min _{\nu \in \Pi\left(\tilde{f}_{1}, \tilde{f}_{2}\right)} \int_{\bar{\Omega} \times \bar{\Omega}}|x-y| d \nu=\int_{\bar{\Omega} \times \bar{\Omega}}|x-y| d \nu_{0},
$$

for some $\nu_{0} \in \Pi\left(\tilde{f}_{1}, \tilde{f}_{2}\right)$. Then, since $\pi_{1} \# \nu_{0}\left\llcorner\partial \Omega=\mathcal{V}^{+}\right.$and $\pi_{2} \# \nu_{0} L \partial \Omega=\mathcal{V}^{-}$,

$$
\int_{\Omega} u_{\infty}\left(f_{+}-f_{-}\right)=\int_{\bar{\Omega} \times \bar{\Omega}}|x-y| d \nu_{0}-\int_{\partial \Omega} g_{1} d \pi_{1} \# \nu_{0}+\int_{\partial \Omega} g_{2} d \pi_{2} \# \nu_{0},
$$

and consequently, since $\nu_{0} \in \mathcal{A}\left(f_{+}, f_{-}\right)$, the above equality together with $(2.7)$ gives

$$
\begin{aligned}
\inf \left\{\int_{\bar{\Omega} \times \bar{\Omega}}|x-y| d \mu-\int_{\partial \Omega} g_{1} d \pi_{1} \# \mu+\int_{\partial \Omega} g_{2} d \pi_{2} \# \mu: \mu \in \mathcal{A}\left(f_{+}, f_{-}\right)\right\} \\
\quad=\int_{\Omega} u_{\infty}\left(f_{+}-f_{-}\right) d x,
\end{aligned}
$$

and the above infimum is in fact a minimum attained at $\nu_{0}$.

The result under condition (1.3) now follows by approximation. Indeed, let $g_{1, n}$ and $g_{2, n}$ be continuous functions on the boundary $\partial \Omega$ satisfying

$$
g_{1, n}(x)-g_{2, n}(y)<|x-y| \quad \forall x, y \in \partial \Omega,
$$

and

$$
g_{i, n} \rightrightarrows g_{i} \quad \text { uniformly on } \partial \Omega, \quad i=1,2
$$


By the previous argument, there exist $u_{\infty, n} \in W^{1, \infty}(\Omega)$, with $\left\|\nabla u_{\infty, n}\right\|_{\infty} \leq 1$ and $g_{1, n} \leq u_{\infty, n} \leq g_{2, n}$ on $\partial \Omega$, and there exist measures $\mu_{n} \in \mathcal{A}\left(f_{+}, f_{-}\right)$satisfying

$$
\begin{aligned}
\int_{\Omega} u_{\infty, n}(x)\left(f_{+}(x)-f_{-}(x)\right) d x & \\
= & \int_{\bar{\Omega} \times \bar{\Omega}}|x-y| d \mu_{n}-\int_{\partial \Omega} g_{1, n} d \pi_{1} \# \mu_{n}+\int_{\partial \Omega} g_{2, n} d \pi_{2} \# \mu_{n} \\
(3.27) & =\min \left\{\int_{\bar{\Omega} \times \bar{\Omega}}|x-y| d \mu-\int_{\partial \Omega} g_{1, n} d \pi_{1} \# \mu+\int_{\partial \Omega} g_{2, n} d \pi_{2} \# \mu: \mu \in \mathcal{A}\left(f_{+}, f_{-}\right)\right\} .
\end{aligned}
$$

By the Morrey-Sobolev embedding theorem and the Arzelà-Ascoli compactness criterion we can suppose that, passing to a subsequence if necessary,

$$
u_{\infty, n} \rightrightarrows u_{\infty} \quad \text { uniformly in } \bar{\Omega} .
$$

Moreover,

$$
\left\|\nabla u_{\infty}\right\|_{\infty} \leq 1, \quad \text { and } \quad g_{1} \leq u_{\infty} \leq g_{2} \text { on } \partial \Omega .
$$

On the other hand, we show that

$$
\mu_{n}(\bar{\Omega} \times \bar{\Omega}) \leq \int_{\Omega}\left(f_{+}(x)+f_{-}(x)\right) d x \quad \forall n \in \mathbb{N} .
$$

Indeed,

$$
\mu_{n}(\bar{\Omega} \times \bar{\Omega})=\int_{\Omega} f_{-}(x) d x+\pi_{2} \# \mu_{n}(\partial \Omega) .
$$

Now, if we define

$$
\tilde{\mu}_{n}:=\mu_{n}-\mu_{n}\llcorner(\partial \Omega \times \partial \Omega),
$$

we have $\tilde{\mu}_{n} \in \mathcal{A}\left(f_{+}, f_{-}\right)$, and

$$
\begin{aligned}
\int_{\bar{\Omega} \times \bar{\Omega}}|x-y| d \tilde{\mu}_{n}- & \int_{\partial \Omega} g_{1} d \pi_{1} \# \tilde{\mu}_{n}+\int_{\partial \Omega} g_{2} d \pi_{2} \# \tilde{\mu}_{n} \\
= & \int_{\bar{\Omega} \times \bar{\Omega}}|x-y| d \mu_{n}-\int_{\partial \Omega} g_{1} d \pi_{1} \# \mu_{n}+\int_{\partial \Omega} g_{2} d \pi_{2} \# \mu_{n} \\
& \quad-\int_{\partial \Omega \times \partial \Omega}\left(|x-y|-g_{1, n}(x)+g_{2, n}(y)\right) d \mu_{n} .
\end{aligned}
$$

Hence, since

$$
|x-y|-g_{1, n}(x)+g_{2, n}(y)>0 \quad \forall x, y \in \partial \Omega,
$$

from (3.27), we deduce that

$$
\mu_{n}(\partial \Omega \times \partial \Omega)=0 .
$$


That is, there is not transportation of mass directly between the boundary, and hence all the mass exported will come from $f_{+}\left(\mathrm{so}, \pi_{2} \# \mu_{n}(\partial \Omega) \leq \int_{\Omega} f_{+}\right)$, and all the mass imported will go to cover $f_{-}\left(\mathrm{so}, \pi_{1} \# \mu_{n}(\partial \Omega) \leq \int_{\Omega} f_{-}\right)$:

$$
\begin{aligned}
\int_{\Omega} f_{+}(x) d x & +\pi_{1} \# \mu_{n}(\partial \Omega) \\
& =\mu_{n}(\bar{\Omega} \times \bar{\Omega}) \geq \mu_{n}(\bar{\Omega} \times \partial \Omega)+\mu_{n}(\partial \Omega \times \bar{\Omega})-\mu_{n}(\partial \Omega \times \partial \Omega) \\
& =\mu_{n}(\bar{\Omega} \times \partial \Omega)+\mu_{n}(\partial \Omega \times \bar{\Omega})=\pi_{2} \# \mu_{n}(\partial \Omega)+\pi_{1} \# \mu_{n}(\partial \Omega),
\end{aligned}
$$

and we get

$$
\pi_{2} \# \mu_{n}(\partial \Omega) \leq \int_{\Omega} f_{+}(x) d x,
$$

(similarly, we get $\left.\pi_{1} \# \mu_{n}(\partial \Omega) \leq \int_{\Omega} f_{-}(x) d x\right)$. Consequently, by (3.29), we obtain (3.28).

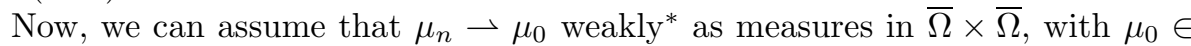
$\mathcal{A}\left(f_{+}, f_{-}\right)$. Then, passing to the limit in (3.27) we conclude the proof.

\subsection{Construction of transport plans via transport maps}

Once a possible pair of export/import masses on the boundary is fixed, call it $\left(\mathcal{V}^{+}, \mathcal{V}^{-}\right)$, and an optimal transport plan $\mu$ is taken for $f_{+} \mathcal{L}^{N}\left\llcorner\Omega+\mathcal{V}^{+}\right.$and $f_{-} \mathcal{L}^{N} L \Omega+\mathcal{V}^{-}$, which can be chosen such that $\mu(\partial \Omega \times \partial \Omega)=0$ because of condition (1.3), we know which part of $f_{+}$, we call it $\tilde{f}_{+}$, is going to be exported and which part of $f_{-}$, we call it $\tilde{f}_{-}$, is covered by imported material,

$$
\tilde{f}_{+}=\pi_{1} \#\left(\mu\llcorner\Omega \times \partial \Omega) \quad \text { and } \quad \tilde{f}_{-}=\pi_{2} \#(\mu\llcorner\partial \Omega \times \Omega) .\right.
$$

Now, we state two facts.

1. Existence of optimal maps. Since $\tilde{f}_{+}$and $\tilde{f}_{-}$are absolutely continuous with respect to the Lebesgue measure, by the Sudakov theorem (see Theorem 6.2 in [2]), there exists an optimal map $t_{2}: \operatorname{supp}\left(\tilde{f}_{+}\right) \rightarrow \partial \Omega$ pushing $\tilde{f}_{+}$forward to $\mathcal{V}^{+}$, and there exists an optimal map $t_{1}: \operatorname{supp}\left(\tilde{f}_{-}\right) \rightarrow \partial \Omega$ an optimal map pushing $\tilde{f}_{-}$ forward to $\mathcal{V}^{-}$. These maps are described by

$$
\begin{aligned}
& g_{2}\left(t_{2}(x)\right)+\left|x-t_{2}(x)\right|=\min _{y \in \partial \Omega}\left(g_{2}(y)+|x-y|\right) \quad \text { for a.e. } x \in \operatorname{supp}\left(\tilde{f}_{+}\right), \\
& g_{1}\left(t_{1}(x)\right)-\left|x-t_{1}(x)\right|=\max _{y \in \partial \Omega}\left(g_{1}(y)-|x-y|\right) \quad \text { for a.e. } x \in \operatorname{supp}\left(\tilde{f}_{-}\right) .
\end{aligned}
$$

Moreover, there exists an optimal map $t_{0}: \operatorname{supp}\left(f_{+}\right) \rightarrow \Omega$ pushing $f_{+}-\tilde{f}_{+}$forward to $f_{-}-\tilde{f}_{-}$.

All these maps are such that the measure defined, for $\varphi \in C(\bar{\Omega} \times \bar{\Omega})$, by

$$
\begin{aligned}
\int_{\bar{\Omega} \times \bar{\Omega}} \varphi(x, y) d \mu^{*}(x, y)= & \int_{\bar{\Omega}} \varphi\left(x, t_{2}(x)\right) \tilde{f}_{+}(x) d x \\
& +\int_{\bar{\Omega}} \varphi\left(x, t_{0}(x)\right)\left(f_{+}(x)-\tilde{f}_{+}(x)\right) d x+\int_{\bar{\Omega}} \varphi\left(t_{1}(y), y\right) \tilde{f}_{-}(y) d y
\end{aligned}
$$


that we write formally as (we will use this formal notation afterwards)

$$
\mu^{*}(x, y)=\tilde{f}_{+}(x) \delta_{y=t_{2}(x)}+\left(f_{+}(x)-\tilde{f}_{+}(x)\right) \delta_{y=t_{0}(x)}+\tilde{f}_{-}(y) \delta_{x=t_{1}(y)},
$$

is an optimal transport plan for our problem, which is given in terms of transport maps.

2. Kantorovich potentials. The limit $u_{\infty}$ is a Kantorovich potential, in the classical sense, for each of the three transport problems that appears in the above description, the transport of $\tilde{f}_{+}$to $\mathcal{V}^{+}$on $\partial \Omega$, of $\mathcal{V}^{-}$on $\partial \Omega$ to $\tilde{f}_{-}$, and of $f_{+}-\tilde{f}_{+}$ to $f_{-}-\tilde{f}_{-}$inside $\Omega$. In fact, there hold

$$
\begin{array}{ll}
u_{\infty}(x)=g_{2}\left(t_{2}(x)\right)+\left|x-t_{2}(x)\right| & \text { for a.e. } x \in \operatorname{supp}\left(\tilde{f}_{+}\right), \\
u_{\infty}(x)=g_{1}\left(t_{1}(x)\right)-\left|x-t_{1}(x)\right| & \text { for a.e. } x \in \operatorname{supp}\left(\tilde{f}_{-}\right), \\
u_{\infty}(x)=u_{\infty}\left(t_{0}(x)\right)+\left|x-t_{0}(x)\right| & \text { for a.e. } x \in \operatorname{supp}\left(f_{+}-\tilde{f}_{+}\right) .
\end{array}
$$

Hence, on the support of the mass that is exported from $\Omega$ and on the support of the mass that is covered by mass imported from $\partial \Omega$, the potential is given only in terms of $g_{2}$ and $g_{1}$, respectively:

$$
\begin{aligned}
& u_{\infty}(x)=\min _{y \in \partial \Omega}\left(g_{2}(y)+|x-y|\right) \quad \text { for a.e. } x \in \operatorname{supp}\left(\tilde{f}_{+}\right), \\
& u_{\infty}(x)=\max _{y \in \partial \Omega}\left(g_{1}(y)-|x-y|\right) \quad \text { for a.e. } x \in \operatorname{supp}\left(\tilde{f}_{-}\right) .
\end{aligned}
$$

To show this claim we argue as follows. Let $t_{0}, t_{1}$ and $t_{2}$ be any optimal transport plans given as above. Then it follows that

$$
\mu^{*}(x, y)=\tilde{f}_{+}(x) \delta_{y=t_{2}(x)}+\left(f_{+}(x)-\tilde{f}_{+}(x)\right) \delta_{y=t_{0}(x)}+\tilde{f}_{-}(y) \delta_{x=t_{1}(y)}
$$

is an optimal transport plan for our problem. Now, we can take, for each $x \in$ $\operatorname{supp}\left(\tilde{f}_{+}\right)$a point $y_{x} \in \partial \Omega$ where $\min _{y \in \partial \Omega}\left(g_{2}(y)+|x-y|\right)$ is attained in such a way that $\tilde{t}_{2}(x)=y_{x}$ is Borel measurable (see for example [5]), and for each $x \in \operatorname{supp}\left(\tilde{f}_{-}\right)$a point $z_{x} \in \partial \Omega$ where $\max _{y \in \partial \Omega}\left(g_{1}(y)-|x-y|\right)$ is attained in such a way that $\tilde{t}_{1}(x)=z_{x}$ is Borel measurable. Then there hold

$$
\begin{aligned}
& g_{2}\left(t_{2}(x)\right)+\left|x-t_{2}(x)\right| \geq g_{2}\left(\tilde{t}_{2}(x)\right)+\left|x-\tilde{t}_{2}(x)\right|, \\
& g_{1}\left(t_{1}(x)\right)-\left|x-t_{1}(x)\right| \leq g_{1}\left(\tilde{t}_{1}(x)\right)-\left|x-\tilde{t}_{1}(x)\right|,
\end{aligned}
$$

and the cost of the transport for the plan

$$
\tilde{f}_{+}(x) \delta_{y=\tilde{t}_{2}(x)}+\left(f_{+}(x)-\tilde{f}_{+}(x)\right) \delta_{y=t_{0}(x)}+\tilde{f}_{-}(y) \delta_{x=\tilde{t}_{1}(y)}
$$

is, in fact, equal to the one for $\mu^{*}$. Hence, the above inequalities are equalities.

On the other hand, by substituting this particular $\mu^{*}$ in (3.5), a careful computation, using that $\left|\nabla u_{\infty}\right| \leq 1$ and that $u_{\infty}=g_{1}$ in $\operatorname{supp}\left(\mathcal{V}^{-}\right)$and $u_{\infty}=g_{2}$ in $\operatorname{supp}\left(\mathcal{V}^{+}\right)$, gives that $u_{\infty}$ is a Kantorovich potential for the transport of $\tilde{f}_{+}$to $\mathcal{V}^{+}$ on $\partial \Omega$, of $\mathcal{V}^{-}$on $\partial \Omega$ to $\tilde{f}_{-}$, and of $f_{+}-\tilde{f}_{+}$to $f_{-}-\tilde{f}_{-}$inside $\Omega$. Alternatively, one can use the Dual Criteria for Optimality and Remark 3.3.

Then, from these facts, we conclude that all the relevant information to build transport maps (transport rays and sets; see [13], [14]) for this problem is encoded by the limit function $u_{\infty}$. 


\section{Examples}

In this section we provide simple examples for which the solution to the mass transport problem described in $\S 2.2$ can be explicitly computed.

Example 4.1. Let $\Omega:=(0,1), f_{+}:=\chi_{(0,1 / 2)}, f_{-}:=\chi_{(3 / 4,1)}, g_{1} \equiv 0$, and $g_{2} \equiv 1 / 2$. For these data the equality (3.5) becomes

$$
\begin{gathered}
\max _{\substack{u \in C([0,1]) \\
u(x)-u(y) \leq x|x-y| \\
0 \leq u(0), u(1) \leq 1 / 2}} \int_{0}^{1 / 2} u(x) d x-\int_{3 / 4}^{1} u(x) d x \\
(4.1)=\min \left\{\int_{[0,1] \times[0,1]}|x-y| d \mu+\frac{1}{2}\left(\pi_{2} \# \mu(0)+\pi_{2} \# \mu(1)\right): \mu \in \mathcal{A}\left(f_{+}, f_{-}\right)\right\} .
\end{gathered}
$$

It is easy to see that the maximum in (4.1) is taken at the function $u_{\infty}$ defined by

$$
u_{\infty}(x)= \begin{cases}x+1 / 2 & \text { if } x \in[0,1 / 4] \\ 1-x & \text { if } x \in[1 / 4,1]\end{cases}
$$

for which

$$
\int_{0}^{1 / 2} u_{\infty}(x) d x-\int_{3 / 4}^{1} u_{\infty}(x) d x=\frac{9}{32} .
$$

Moreover, for $\mu(x, y)=\chi_{(0,1 / 4)}(x) \delta_{y=0}+\chi_{(1 / 4,1 / 2)}(x) \delta_{y=x+1 / 2}$, a simple calculation shows that $\pi_{1} \# \mu=f_{+}$and $\pi_{2} \# \mu=f_{-}+\delta_{0} / 4$, and

$$
\int_{[0,1] \times[0,1]}|x-y| d \mu+\frac{1}{2}\left(\pi_{2} \# \mu(0)+\pi_{2} \# \mu(1)\right)=\frac{9}{32} .
$$

Therefore $\mu$ is a minimizer in (4.1). This optimal plan exports the mass in $(0,1 / 4)$ to the point 0 on the boundary and transports the mass from $(1 / 4,1 / 2)$ to $(1 / 2,1)$.

Observe that importing mass from the point 1 in the boundary is tax free. Nevertheless, to import a little mass from that point would imply exporting more mass to the point 0 where the taxes are sufficiently large to increase the total price of the operation. Nevertheless if we decrease a little bit the taxes on 0 the situation changes. Consider $g_{1} \equiv 0$ and $g_{2} \equiv 1 / 2-b(0<b \leq 1 / 2)$; for these data,

$$
u_{\infty}(x)= \begin{cases}x+1 / 2-b & \text { if } x \in[0,(b+1) / 4], \\ 1-x-b / 2 & \text { if } x \in[(b+1) / 4,1-b / 4], \\ x-1 & \text { if } x \in[1-b / 4,1]\end{cases}
$$

and

$$
\mu(x, y)=\chi_{(0,(b+1) / 4)}(x) \delta_{y=0}+\chi_{((b+1) / 4,1 / 2)}(x) \delta_{y=x+1 / 2-b / 4}+\chi_{(1-b / 4,1)}(y) \delta_{x=1}
$$

realizes the maximum and the minimum in $(3.5)$ with cost $9 / 32-b(b+2) / 10$. Now this optimal plan exports the mass in $(0,(b+1) / 4)$ to the point 0 on the boundary, transports the mass from $((b+1) / 4,1 / 2)$ to $(3 / 4,1-b / 4)$, and imports the mass to cover $(1-b / 4,1)$ from the point 1 on the boundary. 
On the contrary, let us now increase the taxes on 0 . Take $g_{1} \equiv 0, g_{2}(0)=1 / 2+b$ $(0<b \leq 1 / 2)$, and $g_{2}(1)=a(0 \leq a \leq 1)$. In this case,

$$
u_{\infty}(x)= \begin{cases}x+1 / 2+b & \text { if } x \in\left[0,1 / 4-(b-a)^{+} / 2\right], \\ a \wedge b-(x-1) & \text { if } x \in\left[1 / 4-(b-a)^{+} / 2,1\right]\end{cases}
$$

and

$$
\begin{aligned}
& \mu(x, y)=\chi_{\left(0,1 / 4-(b-a)^{+/ 2)}\right.}(x) \delta_{y=0} \\
& \quad+\chi_{\left(1 / 4-(b-a)^{+} / 2,1 / 2-(b-a)^{+/ 2}\right)}(x) \delta_{y=x+1 / 2-(b-a)^{+/ 2}}+\chi_{\left(1 / 2-(b-a)^{+/ 2,1 / 2)}\right.}(x) \delta_{y=1}
\end{aligned}
$$

realizes the maximum and the minimum in (3.5) with total cost $9 / 32+b / 4-$ $\left((b-a)^{+}\right)^{2} / 4$. In this case we are exporting the mass in $\left(0,1 / 4-(b-a)^{+} / 2\right)$ to 0 , transporting the mass in $\left(1 / 4-(b-a)^{+} / 2,1 / 2-(b-a)^{+} / 2\right)$ to $(3 / 4,1)$, and exporting the mass in $\left(1 / 2-(b-a)^{+} / 2,1 / 2\right)$ to 1 .

In the above example the masses $f_{+}$and $f_{-}$do not satisfy the mass balance condition. We now give another example in which the mass balance condition on the masses is satisfied, in order to show the difference between this transport problem and the classical one.

Example 4.2. Let $\Omega:=(0,1), f_{+}:=\chi_{(0,1 / 2)}, f_{-}:=\chi_{(1 / 2,1)}, g_{1} \equiv 0$, and $g_{2} \equiv 1 / 2$. For these data, the maximum and the minimum in (3.5) are taken at

$$
u_{\infty}(x)= \begin{cases}x+1 / 2 & \text { if } x \in[0,1 / 8] \\ -x+3 / 4 & \text { if } x \in[1 / 8,7 / 8] \\ x-1 & \text { if } x \in[7 / 8,1]\end{cases}
$$

and $\mu(x, y)=\chi_{(0,1 / 8)}(x) \delta_{y=0}+\chi_{(1 / 8,1 / 2)}(x) \delta_{y=x+3 / 8}+\chi_{(7 / 8,1)}(y) \delta_{x=1}$, and the cost of this transport problem is $7 / 32$. This optimal plan exports the mass in $(0,1 / 8)$ to the point 0 on the boundary, transports the mass from $(1 / 8,1 / 2)$ to $(1 / 2,7 / 8)$, and imports the mass to cover $(7 / 8,1)$ from the point 1 on the boundary.

This transport problem would have coincided with the classical one if we had put $g_{2} \equiv 1$.

Finally, we give an example in which the import/export taxes coincide.

Example 4.3. Let $\Omega:=(0,1), f_{+}:=\chi_{(0,1 / 2)}, f_{-}:=\chi_{(1 / 2,1)}, g_{1}=g_{2}=g$, $g(0):=0$, and $g(1):=1 / 2$. Now, the maximum and the minimum in (3.5) are taken in

$$
u_{\infty}(x)= \begin{cases}x & \text { if } x \in[0,3 / 8], \\ -x+3 / 4 & \text { if } x \in[3 / 8,5 / 8], \\ x-1 / 2 & \text { if } x \in[5 / 8,1],\end{cases}
$$

and $\mu(x, y)=\chi_{(0,3 / 8)}(x) \delta_{y=0}+\chi_{(3 / 8,1 / 2)}(x) \delta_{y=x+1 / 8}+\chi_{(5 / 8,1)}(y) \delta_{x=1}$, and the total cost of the transport process is $-1 / 32$. This optimal plan exports the mass 
in $(0,3 / 8)$ to the point 0 on the boundary, transports the mass from $(3 / 8,1 / 2)$ to $(1 / 2,5 / 8)$, and imports the mass to cover $(5 / 8,1)$ from the point 1 on the boundary. Observe that in this case the taxes are good enough to get benefits from exporting/importing some mass.

\section{Limited importation/exportation}

In this section we show how to adapt the previous ideas to handle the case in which to the previous setting we add pointwise restrictions on the amount of mass that can be exported/imported.

Now, we only consider on the taxes $T_{i}$ and $T_{e}$ the restriction $T_{i}+T_{e} \geq 0$ on $\partial \Omega$ and we take two functions $M_{i}, M_{e} \in L^{\infty}(\partial \Omega)$ with $M_{i}, M_{e} \geq 0$ that are going to represent the limitations on importation/exportation mass across the boundary. Accordingly, consider that the businessman has the restriction of limiting the amount of mass by the pointwise quantities of $M_{e}(x)$ for export at $x \in \partial \Omega$ and $M_{i}(x)$ for import at $x$ on the boundary. We then need to impose

$$
-\int_{\partial \Omega} M_{e} \leq-\int_{\Omega}\left(f_{+}-f_{-}\right) \leq \int_{\partial \Omega} M_{i},
$$

that says that the interplay with the boundary (that is, importation/exportation) is possible. Now, the main goal is, given the new set

$$
\mathcal{A}_{\ell}\left(f_{+}, f_{-}\right):=\left\{\begin{array}{ll}
\mu \in \mathcal{M}^{+}(\bar{\Omega} \times \bar{\Omega}): & \pi_{1} \# \mu\left\llcorner\Omega=f_{+} \mathcal{L}^{N}\llcorner\Omega,\right. \\
& \pi_{2} \# \mu\left\llcorner\Omega=f_{-} \mathcal{L}^{N}\llcorner\Omega,\right. \\
& \pi_{1} \# \mu\left\llcorner\partial \Omega \leq M_{i}, \pi_{2} \# \mu\left\llcorner\partial \Omega \leq M_{e}\right.\right.
\end{array}\right\},
$$

to obtain

$$
\min \left\{\int_{\bar{\Omega} \times \bar{\Omega}}|x-y| d \mu+\int_{\partial \Omega} T_{i} d\left(\pi_{1} \# \mu\right)+\int_{\partial \Omega} T_{e} d\left(\pi_{2} \# \mu\right): \mu \in \mathcal{A}_{\ell}\left(f_{+}, f_{-}\right)\right\} .
$$

In this situation, the dual problem proposed by the clever fellow is similar to the previous one but with the following new conditions on the payments $\varphi$ and $\psi$. The fellow proposes that he will undertake the transaction in such a way that (2.4) is satisfied, but now his charges will not necessarily satisfy (here is the main difference with the previous case) (2.5). Nevertheless he will pay the following compensation when $\varphi<-T_{i}$ or $\psi<-T_{e}$ on the boundary, in the amount of

$$
\int_{\partial \Omega} M_{i}\left(-\varphi-T_{i}\right)^{+}+\int_{\partial \Omega} M_{e}\left(-\psi-T_{e}\right)^{+} .
$$

We introduce the new functional $J_{\ell}: C(\bar{\Omega}) \times C(\bar{\Omega}) \rightarrow \mathbb{R}$, defined by

$$
J_{\ell}(\varphi, \psi):=\int_{\Omega} \varphi f_{+}+\int_{\Omega} \psi f_{-}-\int_{\partial \Omega} M_{i}\left(-\varphi-T_{i}\right)^{+}-\int_{\partial \Omega} M_{e}\left(-\psi-T_{e}\right)^{+},
$$


and the set

$$
\mathcal{B}_{\ell}:=\{(\varphi, \psi) \in C(\bar{\Omega}) \times C(\bar{\Omega}): \varphi(x)+\psi(y) \leq|x-y|\} .
$$

Then the aim of the fellow is to obtain

$$
\sup _{(\varphi, \psi) \in \mathcal{B}_{\ell}} J_{\ell}(\varphi, \psi)
$$

In this situation we also get

$$
\begin{aligned}
& \sup _{(\varphi, \psi) \in \mathcal{B}_{\ell}} J_{\ell}(\varphi, \psi) \\
& \quad \leq \inf _{\mu \in \mathcal{A}_{\ell}\left(f_{+}, f_{-}\right)}\left\{\int_{\bar{\Omega} \times \bar{\Omega}}|x-y| d \mu+\int_{\partial \Omega} T_{i} d\left(\pi_{1} \# \mu\right)+\int_{\partial \Omega} T_{e} d\left(\pi_{2} \# \mu\right)\right\},
\end{aligned}
$$

and again we can show that there is no gap between the costs. In fact, if we now consider the energy functional

$$
\Psi_{\ell}(u):=\int_{\Omega} \frac{|\nabla u(x)|^{p}}{p} d x-\int_{\Omega} f(x) u(x) d x+\int_{\partial \Omega} j(x, u(x)),
$$

where

$$
j(x, r)= \begin{cases}M_{i}(x)\left(-T_{i}(x)-r\right) & \text { if } r<-T_{i}(x) \\ 0 & \text { if }-T_{i}(x) \leq r \leq T_{e}(x) \\ M_{e}(x)\left(r-T_{e}(x)\right) & \text { if } r>T_{e}(x)\end{cases}
$$

the variational problem

$$
\min _{u \in W^{1, p}(\Omega)} \Psi_{\ell}(u)
$$

has a minimizer $u_{p}$ in $W^{1, p}(\Omega)$, which is a least energy solution (in an adequate sense; see (5.5)) of the nonlinear boundary problem

$$
\begin{cases}-\Delta_{p} u=f & \text { in } \Omega \\ |\nabla u|^{p-2} \nabla u \cdot \eta+\partial j(\cdot, u(\cdot)) \ni 0 & \text { on } \partial \Omega .\end{cases}
$$

Then, we have the following result:

Theorem 5.1. Given the solutions $u_{p}$ to (5.1), there is a sequence $p_{i} \rightarrow \infty$ such that $u_{p_{i}} \rightarrow u_{\infty}$ uniformly. Moreover, $u_{\infty}$ is a maximizer of the variational problem

$$
\max \left\{\int_{\Omega} w(x) f(x) d x-\int_{\partial \Omega} j(x, w(x)): w \in W^{1, \infty}(\Omega),\|\nabla w\|_{L^{\infty}(\Omega)} \leq 1\right\} .
$$

Proof. The proof is analogous to the proof of Theorem 3.1. Note that here we do not need to impose a condition like (1.3), since to obtain $u_{p}$ we are minimizing over all of $W^{1, p}(\Omega)$ without any pointwise constraint, therefore we can use any $w$ with $\|\nabla w\|_{L^{\infty}(\Omega)} \leq 1$ as a test to obtain the required uniform bounds for $\left\|\nabla u_{p}\right\|_{L^{p}(\Omega)}$.

Finally, we observe that to show (5.2) we use the fact that $j(x, \cdot)$ is lower semicontinuous. 
Theorem 5.2. Assume that $T_{i}$ and $T_{e}$ satisfy $T_{i}(x)+T_{e}(x) \geq 0$ on $\partial \Omega$. Then, there holds the duality result

$$
\begin{aligned}
& \int_{\Omega} u_{\infty}(x)\left(f_{+}(x)-f_{-}(x)\right) d x-\int_{\partial \Omega} j\left(x, u_{\infty}(x)\right) \\
& =\sup \left\{J_{\ell}(\varphi, \psi):(\varphi, \psi) \in \mathcal{B}_{\ell}\right\} \\
(5.3)= & \min \left\{\int_{\bar{\Omega} \times \bar{\Omega}}|x-y| d \mu+\int_{\partial \Omega} T_{i} d \pi_{1} \# \mu+\int_{\partial \Omega} T_{e} d \pi_{2} \# \mu: \mu \in \mathcal{A}_{\ell}\left(f_{+}, f_{-}\right)\right\} .
\end{aligned}
$$

Remark 5.3. Fix $\mu \in \mathcal{A}_{\ell}\left(f_{+}, f_{-}\right)$a measure at which the minimum in (5.3) is assumed. If $\mu_{i}:=\pi_{i} \# \mu, i=1,2$, by the Kantorovich-Rubinstein theorem, we have

$$
\left\{\int_{\bar{\Omega} \times \bar{\Omega}}|x-y| d \nu: \nu \in \Pi\left(\mu_{1}, \mu_{2}\right)\right\}=\max \left\{\int_{\bar{\Omega}} u d\left(\mu_{1}-\mu_{2}\right): u \in K_{1}(\bar{\Omega})\right\} .
$$

Following the lines of Remark 3.3 we have that $\mu$ is an optimal transport plan for (5.4), and $u_{\infty}$ is a Kantorovich potential for (5.4). Moreover, $u_{\infty} \leq-T_{i}$ on $\operatorname{supp}\left(\mu_{1} L \partial \Omega\right)$ and $u_{\infty} \geq T_{e}$ on $\operatorname{supp}\left(\mu_{2}\llcorner\partial \Omega)\right.$.

The proof of Theorem 5.2 uses the following result and an approximation argument for the case $T_{i}(x)+T_{e}(x) \geq 0$ similar to that given for Theorem 3.1.

Theorem 5.4. Assume that $T_{i}$ and $T_{e}$ satisfy

$$
T_{i}(x)+T_{e}(x)>0 \quad \forall x \in \partial \Omega .
$$

Let $u_{p}$ be a minimizer of the problem (5.1). Then:

(1) There exist $\mathcal{X}_{p} \cdot \eta \in L^{\infty}(\partial \Omega),-\mathcal{X}_{p} \cdot \eta \in \partial j\left(x, u_{p}\right)$ a.e. $x \in \partial \Omega$ such that

$$
\int_{\partial \Omega} \mathcal{X}_{p} \cdot \eta \varphi=\int_{\Omega}\left|D u_{p}\right|^{p-2} D u_{p} \cdot \nabla \varphi-\int_{\Omega} f \varphi \quad \text { for all } \varphi \in W^{1, p}(\Omega) .
$$

There exists a sequence $p_{i} \rightarrow+\infty$ such that $\mathcal{X}_{p_{i}} \cdot \eta$ converges weakly* in $L^{\infty}(\partial \Omega)$ to a function $\mathcal{V} \in L^{\infty}(\partial \Omega)$ with $\mathcal{V}^{+} \leq M_{i}$ and $\mathcal{V}^{-} \leq M_{e}$.

(2) $u_{\infty}$ is a Kantorovich potential for the classical transport problem for the measures $f_{+} \mathcal{L}^{N}\left\llcorner\Omega+\mathcal{V}^{+} d \mathcal{H}^{N-1}\left\llcorner\partial \Omega\right.\right.$ and $f_{-} \mathcal{L}^{N}\left\llcorner\Omega+\mathcal{V}^{-} d \mathcal{H}^{N-1}\llcorner\partial \Omega\right.$.

Proof. The proof is similar to that of Theorem 3.4. Again, $u_{\infty}$ is a Kantorovich potential for the new transport problem, and moreover, the variational approach provides the required import/export masses on the boundary, $\mathcal{V}^{+}$and $\mathcal{V}^{-}$.

The only difference occurs when performing the following computations (we use here the same notations as in the proof of Theorem 3.4).

We have that $\mathcal{X}_{p} \cdot \eta$ is a Radon measure with

$$
\operatorname{supp}\left(\left(\mathcal{X}_{p} \cdot \eta\right)^{+}\right) \subset\left\{x \in \partial \Omega: u_{p}(x) \leq-T_{i}(x)\right\},
$$

and

$$
\operatorname{supp}\left(\left(\mathcal{X}_{p} \cdot \eta\right)^{-}\right) \subset\left\{x \in \partial \Omega: u_{p}(x) \geq T_{e}(x)\right\} .
$$


We show that

$$
\left(\mathcal{X}_{p} \cdot \eta\right)^{+} \leq M_{i}
$$

and

$$
\left(\mathcal{X}_{p} \cdot \eta\right)^{-} \leq M_{e}
$$

For a positive smooth function $\varphi$ we have

$$
\left\langle\mathcal{X}_{p} \cdot \eta, \varphi\right\rangle \geq \limsup _{t \rightarrow 0^{+}}-\int_{\partial \Omega} \frac{j\left(x, u_{p}+t \varphi\right)-j\left(x, u_{p}\right)}{t} .
$$

Now, we observe that

$$
\begin{aligned}
-\int_{\partial \Omega} \frac{j\left(x, u_{p}+t \varphi\right)-j\left(x, u_{p}\right)}{t} & M_{e} \varphi \\
\geq & -\int_{\left\{x \in \partial \Omega: u_{p}(x) \geq T_{e}(x)\right\}} M_{e}\left(u_{p}+t \varphi-T_{e}\right), \\
& -\frac{1}{t} \int_{\left\{x \in \partial \Omega: u_{p}(x)+t \varphi(x)>T_{e}(x)>u_{p}(x)\right\}} M_{e} \varphi \\
\geq & -\int_{\left\{x \in \partial \Omega: u_{p}(x) \geq T_{e}(x)\right\}} M_{e} \rightarrow-\int_{\left\{x \in \partial \Omega: u_{p}(x) \geq T_{e}(x)\right\}} M_{e} \varphi .
\end{aligned}
$$

Hence,

$$
\mathcal{X}_{p} \cdot \eta \geq-M_{e} \chi_{\left\{u_{p} \geq T_{e}\right\}}, \quad \text { so } \quad\left(\mathcal{X}_{p} \cdot \eta\right)^{-} \leq M_{e} \chi_{\left\{u_{p} \geq T_{e}\right\}}
$$

Since we have (5.6), we get (5.8). Similarly, we obtain (5.7).

Consequently, we have that (5.5) holds true.

Taking $\varphi=u_{p}$ in (5.5) we get, taking into account the $L^{\infty}$-boundedness of $u_{p}$, (5.7), and (5.8), that there exists a constant $C$ such that

$$
\int_{\Omega}\left|\nabla u_{p}\right|^{p}=\int_{\Omega} f u_{p}+\int_{\partial \Omega} \mathcal{X}_{p} \cdot \eta u_{p} \leq C .
$$

That is the measures $\mathcal{X}_{p} \mathcal{L}^{N} L \Omega$ are equibounded in $\Omega$. Therefore, there exists a sequence $p_{i} \rightarrow \infty$ such that

$$
\begin{array}{cl}
u_{p_{i}} \rightrightarrows u_{\infty} & \text { uniformly in } \bar{\Omega}, \quad \text { with }\left\|\nabla u_{\infty}\right\|_{\infty} \leq 1, \\
\mathcal{X}_{p_{i}} \rightarrow \mathcal{X} & \text { weakly }^{*} \text { as measures in } \Omega,
\end{array}
$$

and

$$
\mathcal{X}_{p_{i}} \cdot \eta \rightarrow \mathcal{V} \quad \text { weakly }^{*} \text { in } L^{\infty}(\partial \Omega) .
$$

Moreover, we have that

$$
\int_{\Omega} \nabla \varphi d \mathcal{X}=\int_{\Omega} f \varphi d x+\int_{\partial \Omega} \mathcal{V} \varphi \quad \forall \varphi \in C^{1}(\bar{\Omega}) .
$$


That is, formally,

$$
\left\{\begin{aligned}
-\operatorname{div}(\mathcal{X}) & =f & & \text { in } \Omega \\
\mathcal{X} \cdot \eta & =\mathcal{V} & & \text { on } \partial \Omega .
\end{aligned}\right.
$$

From this point the proof is the same as that of Theorem 3.4.

Note that the proof of the approximation argument in this case is even simpler since the measures $\mathcal{V}^{+}$and $\mathcal{V}^{-}$are uniformly bounded by $\left\|M_{i}\right\|_{\infty}$ and $\left\|M_{e}\right\|_{\infty}$ on $\partial \Omega$.

We remark that we have taken $M_{i}$ and $M_{e}$ to be $L^{\infty}$-functions only for simplicity.

Finally, we give a simple example in which the limit on the export/import mass increases the total cost and modifies the Kantorovich potential and the optimal transport plan.

Example 5.5. Let $\Omega:=(0,1), f_{+}:=\chi_{(0,1 / 2)}, f_{-}:=\chi_{(1 / 2,1)}, g_{1} \equiv 0$, and $g_{2} \equiv 1 / 2$. For the first case studied, in which we do not limit the amount of mass that enters or leaves the domain ( say $M_{i}=M_{e}=+\infty$ ), in Example 4.2 we explicitly compute that the cost of the transport problem is $56 / 16^{2}$ and that it is obtained with the Kantorovich potential

$$
u_{\infty}(x)= \begin{cases}x+1 / 2 & \text { if } x \in[0,1 / 8] \\ -x+3 / 4 & \text { if } x \in[1 / 8,7 / 8] \\ x-1 & \text { if } x \in[7 / 8,1]\end{cases}
$$

in this case an optimal transport plan is

$$
\mu(x, y)=\chi_{(0,1 / 8)}(x) \delta_{y=0}+\chi_{(1 / 8,1 / 2)}(x) \delta_{y=x+3 / 8}+\chi_{(7 / 8,1)}(y) \delta_{x=1} .
$$

Now, we consider the limiting functions $M_{i}=M_{e}=1 / 16$. In this case the transport cost is $58 / 16^{2}$. It is attained at

$$
u_{\infty}(x)= \begin{cases}x+5 / 8 & \text { if } x \in[0,1 / 16] \\ -x+3 / 4 & \text { if } x \in[1 / 16,15 / 16] \\ x-9 / 8 & \text { if } x \in[15 / 16,1]\end{cases}
$$

and an optimal transport plan is given by

$$
\mu(x, y)=\chi_{(0,1 / 16)}(x) \delta_{y=0}+\chi_{(1 / 16,1 / 2)}(x) \delta_{y=x+7 / 16}+\chi_{(15 / 16,1)}(y) \delta_{x=1} .
$$

\section{References}

[1] Aguen, M.: Existence of solutions to degenerate parabolic equations via the MongeKantorovich theory. Adv. Differential Equations 10 (2005), no. 3, 309-360.

[2] Ambrosio, L.: Lecture notes on optimal transport problems. In Mathematical aspects of evolving interfaces (Funchal, 2000), 1-52. Lecture Notes in Math. 1812, Springer, Berlin, 2003. 
[3] Ambrosio, L., Gigli, N. And Savaré, G.: Gradient flows in metric spaces and in the space of probability measures. Lectures in Mathematics ETH Zürich, Birkhäuser Verlag, Basel, 2005.

[4] Ambrosio, L. And Pratelli, A.: Existence and stability results in the $L^{1}$ theory of optimal transportation. In Optimal transportation and applications (Martina Franca, 2001), 123-160. Lecture Notes in Math. 1813, Springer, Berlin, 2003.

[5] Brown, L. D. And Purves, R.: Measurable selections of extrema. Ann. Statist. 1 (1973), 902-912.

[6] Caffarelli, L. A. And McCann, R. J.: Free boundaries in optimal transport and Monge-Ampère obstacle problems. Ann. of Math. (2) 171 (2010), no. 2, 673-730.

[7] Evans, L. C. And Gangbo, W.: Differential equations methods for the MongeKantorovich mass transfer problem. Mem. Amer. Math. Soc. 137 (1999), no. 653, viii+66 pp.

[8] Figalli, A.: The optimal partial transport problem. Arch. Ration. Mech. Anal. 195 (2010), no. 2, 533-560.

[9] Figalli, A. And Gigli, N.: A new transportation distance between non-negative measures, with applications to gradients flows with Dirichlet boundary conditions. J. Math. Pures Appl. (9) 94 (2010), no. 2, 107-130.

[10] Kantorovich, L. V.: On the translocation of masses. C.R. (Doklady) Acad. Sci. URSS (N.S.) 37 (1942), 199-201.

[11] Sudakov, V. N.: Geometric problems in the theory of infinite-dimensional probability distributions. Cover to cover translation of Trudy Mat. Inst. Steklov 141 (1976). Proc. Stekelov Inst. Math. 1979, 1-178.

[12] Talenti, G.: Inequalities in rearrangement invariant function spaces. In Nonlinear analysis, function spaces and applications, Vol. 5 (Prague, 1994), 177-230. Prometheus, Prague, 1994.

[13] Villani, C.: Topics in optimal transportation. Graduate Studies in Mathematics 58, American Mathematical Society, Providence, RI, 2003.

[14] Villani, C.: Optimal transport. Old and new. Fundamental Principles of Mathematical Sciences 338, Springer-Verlag, Berlin, 2009.

Received May 10, 2012.

José M. Mazón: Departament d'Anàlisi Matemàtica, Universitat de València, Dr. Moliner 50, 46100-Burjassot, Valencia, Spain.

E-mail: mazon@uv.es

Julio D. Rossi: Departamento de Análisis Matemático, Universidad de Alicante, Apdo. correos 99, 03080-Alicante, Spain; and Departamento de Matemática, FCEyN UBA, Ciudad Universitaria, Pab 1 (1428), Buenos Aires, Argentina.

E-mail: jrossi@dm.uba.ar

Julián Toledo: Departament d'Anàlisi Matemàtica, Universitat de València, Dr. Moliner 50, 46100-Burjassot, Valencia, Spain.

E-mail: toledojj@uv.es

J. M. M. and J. T. have been partially supported by the Spanish MEC and FEDER, project MTM2012-31103. J. D. R. has been partially supported by MEC MTM2010-18128 (Spain). 